\title{
Experimental design for estimating unknown hydraulic conductivity in an aquifer using a genetic algorithm and reduced order model
}

\author{
Timothy T. Ushijima \\ Department of Civil and Environmental Engineering, University of California, Los Angeles, \\ California, 90095, USA. \\ E-mail: tim.ushijima@gmail.com \\ William W.-G. Yeh (Corresponding Author) \\ Department of Civil and Environmental Engineering, University of California, Los Angeles, \\ California, 90095, USA. \\ Tel.: +1 3108252300 \\ Fax: +13108257581 \\ E-mail: williamy@seas.ucla.edu \\ Keywords: Model reduction, Proper orthogonal decomposition, Robust experimental design, \\ Max-min optimization, Genetic algorithm
}

\begin{abstract}
We develop an experimental design algorithm to select locations for a network of observation wells that provide the maximum robust information about unknown hydraulic conductivity in a confined, anisotropic aquifer. Since the information that a design provides is dependent on an aquifer's hydraulic conductivity, a robust design is one that provides the maximum information in the worst-case scenario. The design can be formulated as a max-min optimization problem. The problem is generally non-convex, non-differentiable, and contains integer variables. We use a Genetic Algorithm (GA) to perform the combinatorial search. We employ proper orthogonal decomposition (POD) to reduce the dimension of the groundwater model, thereby reducing the computational burden posed by employing a GA. The GA algorithm exhaustively searches for the robust design across a set of hydraulic conductivities and finds an approximate design (called the High Frequency Observation Well Design) through a Monte Carlo-type search. The results
\end{abstract}


from a small-scale 1-D test case validate the proposed methodology. We then apply the methodology to a realistically-scaled 2-D test case. 


\section{Introduction}

\section{1.a. Background}

Hydraulic conductivity is one of the most important parameters to take into account when modeling an aquifer. Unknown hydraulic conductivities can have drastic effects on the reliability of groundwater model results. Estimating these unknown hydraulic conductivities through inverse modeling is an important area of research in groundwater modeling. Solving the inverse problem requires field observations of head. However, collecting good observations is expensive, time consuming, and difficult, particularly if aquifer parameters vary spatially. As a result, inverse modeling always faces an observation scarcity problem. Given an infinite budget, we could take observations at infinitesimally fine spatial and temporal resolutions; however, in the real world there are budget constraints on the number of experiments (observations) that can be conducted. In general, the goal of optimal experimental design for parameter estimation is to select the observation locations and sampling frequency such that a specified criterion is optimized subject to a set of constraints. The constraints frequently encountered are cost, reliability of the estimated parameters, and time and duration of the experiments. In groundwater modeling, if one assumes that observations are taken from the beginning of the pumping test to the end, the experimental design problem is simplified to the determination of a network of observation locations. The most commonly used criterion for optimal experimental design is the maximization of a measure of the information matrix. This formulation generally lends itself to a combinatorial optimization problem that is nonlinear and non-convex. We then can determine the tradeoff between number of experiments and amount of useful information collected by varying the number of experiments and evaluating the amount of useful information received for each experiment. While the concept of experimental design for parameter estimation in the 
context of groundwater modeling has been investigated extensively, the combinatorial search required to solve for the optimal observation network of observations has challenged many studies. A realistic, highly discretized, large-scale groundwater model, referred to in this paper as the full model, may have tens or hundreds of thousands of nodes and a resulting equivalent number of equations. As a consequence, the dimension of the combinatorial search quickly becomes so large that it is infeasible to solve through mathematical programming techniques such as mixed integer nonlinear programming. Thus other methods are required to solve this optimization problem. Genetic Algorithms (GAs) are one of a number of methods that have been developed over the years to solve large-scale optimization problems that are difficult or impossible to solve through traditional mathematical programming techniques. GAs do not require the calculation of derivatives and also can deal with discontinuous functions. While GAs have been used by a number of studies to design optimal observation networks using various optimality criteria [1-3], many of these studies were challenged by the fact that GAs require a large number of model calls. Thus for a highly discretized groundwater model, using a GA to design an optimal observation network may be computationally inefficient, even infeasible. To overcome this, we apply a proper orthogonal decomposition (POD) model reduction to the groundwater model to reduce its spatial dimension. POD is an effective model reduction technique that maintains the physics of the groundwater model. In many instances it has been shown that the reduced model is orders of magnitudes smaller than the original full model and runs 1,000 times faster. Since the parameters themselves are to be estimated, we develop a parameter-independent reduced model for the optimal experimental design. By doing this, we are able to reduce an inordinately large-scale, combinatorial optimization problem to a manageable size. 
Ushijima and Yeh [4] applied POD model reduction to develop an algorithm for experimental design that seeks information related to unknown pumping rates. The current study expands on this previous study by applying POD model reduction to develop an algorithm for experimental design that seeks information related to unknown model parameters. While similar, these two problems are different. Because the response of the groundwater system is linear with respect to changes in pumping, the sensitivity coefficients are invariant and only need to be calculated once. On the other hand, the response of the groundwater system to changes in hydrologic parameters (e.g. hydraulic conductivity) is nonlinear and dependent on the unknown parameters. This makes the current study much more challenging and difficult.

The major contribution of this paper is to demonstrate the applicability of using reduced order models to ensure that solving the underlying combinatorial optimization problem is computationally feasible. In other words, our proposed method allows us to solve realisticallyscaled, optimal experimental design for parameter estimation problems in groundwater modeling that were not possible to solve in the past.

\section{1.b. Confined Aquifer Groundwater Model}

The governing equation for three-dimensional groundwater flow in a confined, anisotropic aquifer, can be expressed by the following partial differential equation (PDE) [5]:

$$
\frac{\partial}{\partial x}\left(K_{x} \frac{\partial h}{\partial x}\right)+\frac{\partial}{\partial y}\left(K_{y} \frac{\partial h}{\partial y}\right)+\frac{\partial}{\partial z}\left(K_{z} \frac{\partial h}{\partial z}\right)-F=S_{s} \frac{\partial h}{\partial t}
$$

with initial and boundary conditions 


$$
\begin{gathered}
h(x, y, z)=f_{1}(x, y, z) \\
h(x, y, z, t)=f_{2}(x, y, z, t), \quad(x, y, z, t) \in\left(\Gamma_{1}\right) \\
q_{n}(x, y, z, t)=f_{3}(x, y, z, t), \quad(x, y, z, t) \in\left(\Gamma_{2}\right)
\end{gathered}
$$

where $h$ is the hydraulic head [L]; $K_{x}, K_{y}$, and $K_{z}$ are the hydraulic conductivities in the $x, y$, and $z$ directions $[\mathrm{L} / \mathrm{T}] ; S_{s}$ is the specific storage $\left[\mathrm{L}^{-1}\right] ; F$ is the specific volumetric pumping rate $\left[\mathrm{T}^{-1}\right] ; q_{n}$ is the specific discharge normal to the flux boundary $\left(\Gamma_{2}\right)\left[\mathrm{T}^{-1}\right] ; \Gamma_{1}$ is the fixed head boundary; $f_{1}, f_{2}$, and $f_{3}$ are known functions; L denotes the length unit (meters, feet, etc.); and T denotes the time unit (days, hours, etc.). Without loss of generality we can change the state variable from head $(\boldsymbol{h})$ to drawdown $(\boldsymbol{s})$. Drawdown is defined as the difference between the initial head $(\boldsymbol{H})$ and head at some time $(\boldsymbol{h})$ (i.e. $\boldsymbol{s}=\boldsymbol{H}-\boldsymbol{h})$. This linear transformation converts the initial and Dirichlet boundary conditions to zero. The governing PDE (Eq. (1)) then can be discretized through finite difference (or finite element) approximations into a system of linear ordinary differential equations (ODEs):

$$
\boldsymbol{B} \frac{d s}{d t}+A s=q
$$

where $\boldsymbol{s} \in R^{N_{n}}$ is a vector of drawdown values, $\boldsymbol{A} \in R^{N_{n} x N_{n}}$ is the stiffness matrix, $\boldsymbol{B} \in R^{N_{n} \times N_{n}}$ is the mass matrix, $\boldsymbol{q} \in R^{N_{n}}$ is a vector of sinks (pumping, recharge, evaporation, etc.), and $N_{n}$ is the total number of nodes the aquifer is discretized into. Eq. (2) is referred to as the full model. By approximating the time derivative and assuming that all model parameters except hydraulic conductivity are fixed, we can write this linear set of ODEs as a set of linear equations:

$$
A(k) s=b
$$


where $\boldsymbol{A}(\boldsymbol{k}) \in R^{N_{n} \times N_{n}}$ contains the model parameters as well as initial and boundary conditions, $\boldsymbol{b} \in R^{N_{n}}$ contains the forcing, and $\boldsymbol{k}$ is a vector of hydraulic conductivities. Because of the dimension of $\boldsymbol{A}(\boldsymbol{k})$, solving (3) is very computationally expensive. To overcome this, we use Proper Orthogonal Decomposition (POD) to reduce the dimension of the groundwater model and thus alleviate the computation burden of solving the full model.

\section{1.c. Proper Orthogonal Decomposition (POD)}

Many studies have focused on POD model reduction [6-8] as well as its use in reducing groundwater models [9-13]. POD projects a system of linear equations of dimension $N_{n}$ onto a subspace of dimension $n_{p}$ such that $n_{p} \leq N_{n}$ and $\hat{\boldsymbol{s}}=\boldsymbol{P r}$, where $\hat{\boldsymbol{s}} \in R^{N_{n}}$ is the approximation of the original state vector $(s), \boldsymbol{P} \in R^{N_{n} \times n_{p}}$ is a the projection operator given by a matrix whose columns form an orthogonal basis spanning the subspace $V \subset R^{N_{n}}$, and $\boldsymbol{r} \in R^{n_{p}}$ is a vector of the reduced solution [11]. We construct the matrix $\boldsymbol{P}$ by collecting snapshots of the drawdown from the full model at various times and storing the snapshots in the matrix $\boldsymbol{X} \in R^{N_{n} x n_{s p}}$ where $n_{s p}$ is the number of snapshots taken. We use the method developed by Siade et al. [11] to approximate the optimal times to take snapshots so that the model accurately represents the dynamics of the original system. Vermeulen et al. [9] showed that taking snapshots at all combinations of hydraulic conductivity results in a parameter-independent reduced model guaranteeing that for any $\boldsymbol{k}$, the error $(\boldsymbol{e}=\boldsymbol{P r}-\boldsymbol{s})$ will be less than a specified tolerance level

$(\tau)$. However, this combinatorial search would require an inordinate number of full model calls. To minimize the number of model calls, we use a greedy algorithm like the one proposed by 
Grepl and Patera [14] to choose hydraulic conductivity combinations with which to take snapshots based on the residual $\left(\boldsymbol{r}_{e}=\boldsymbol{A}(\boldsymbol{k}) \boldsymbol{P r}-\boldsymbol{b}\right)$. Note that the norm of the residual $\left(\boldsymbol{r}_{e}\right)$ can be computed completely in the reduced space without running the full model. It has been shown that the residual $\left(\boldsymbol{r}_{e}\right)$ can be used to estimate the true error $(\boldsymbol{e})$, which is defined as the difference between the solution of the full model and solution of the reduced model [14-17]. However, Hasenauer et al. [17] pointed-out that in many cases $\left\|\boldsymbol{r}_{e}\right\|_{2} \square\|\boldsymbol{e}\|_{2}$. Hinze and Kunkel [18] developed a method to linearly scale the residual given a one-dimensional parameter space. Pasetto et al. [13] extended this concept by employing a non-linear scaling factor such that

$$
d\left\|\boldsymbol{r}_{e}\right\|_{2}=\|\boldsymbol{e}\|_{2}
$$

where $d$ is the scaling factor. This scaled residual is then used to choose hydraulic conductivity combinations. Choosing hydraulic conductivity combinations based on the norm of the error allows us to estimate the lower bound of the accuracy of the reduced model and the reduced model's ability to approximate the Jacobian matrix. We use the algorithm proposed by Pasetto et al. [13] to select hydraulic conductivity combinations to construct a parameter-independent reduced model. The algorithm combines a greedy algorithm for snapshot selection in the parameter space and an approximation of the optimal distribution of the snapshots in time. Using a residual-based estimation of the error associated with the reduced model allows a considerable reduction of the number of full model calls required for the computation of the principal vectors.

Assuming we know what snapshots to collect and store in $\boldsymbol{X}$, we perform singular value decomposition (SVD) on $\boldsymbol{X}$ such that

$$
\boldsymbol{X}=\boldsymbol{P} \Sigma \boldsymbol{V}^{T}
$$


where $\boldsymbol{P} \in R^{N_{n} x n_{p}}$ and $\boldsymbol{V} \in R^{\mathrm{n}_{p} x n_{p}}$ contain the left and right singular vectors of $\boldsymbol{X}$, respectively, and $\boldsymbol{\Sigma} \in R^{\mathrm{n}_{p} \times n_{p}}$ is a diagonal matrix containing the singular values of $\boldsymbol{X}$ [19]. In previous work $\boldsymbol{P}$ was constructed by finding the eigenvectors of $\boldsymbol{X} \boldsymbol{X}^{T}\left(\boldsymbol{X} \boldsymbol{X}^{T}=\boldsymbol{Q} \boldsymbol{\Lambda} \boldsymbol{Q}^{-1}\right)$ and retaining the $n_{p}$ principal vectors of $\boldsymbol{Q}$ [11] where $\boldsymbol{Q} \in R^{N_{n} \times n_{p}}$ is a matrix containing the eigenvectors of $\boldsymbol{X}$ and $\boldsymbol{\Lambda} \in R^{N_{n} x N_{n}}$ is a matrix containing the eigenvalues of $\boldsymbol{X}$. Since $\operatorname{Range}(\boldsymbol{P})=\operatorname{Range}(\boldsymbol{Q})$, to be consistent with previous work we refer to $\boldsymbol{P}$ as a matrix containing the principal vectors of $\boldsymbol{X}$. We then use the Galerkin projection to reduce the full model (Eq. (2)) as follows [11]:

$$
\boldsymbol{P}^{T} \boldsymbol{B P} \frac{d \boldsymbol{r}}{d t}+\boldsymbol{P}^{T} \boldsymbol{A P r}=\boldsymbol{P}^{T} \boldsymbol{q}
$$

Alternatively, we can reduce the model by projecting Eq. (3) as follows:

$$
\boldsymbol{P}^{T} \boldsymbol{A}(\boldsymbol{k}) \boldsymbol{P r}=\boldsymbol{P}^{T} \boldsymbol{b}
$$

Eq. (5) can be transformed into Eq. (6) by approximating the time derivative in Eq. (5). If we let $\boldsymbol{P}^{T} \boldsymbol{A}(\boldsymbol{k}) \boldsymbol{P}=\tilde{\boldsymbol{A}}(\boldsymbol{k})$ and $\boldsymbol{P}^{T} \boldsymbol{b}=\tilde{\boldsymbol{b}}$, Eq. (5) becomes

$$
\tilde{\boldsymbol{A}}(\boldsymbol{k}) \boldsymbol{r}=\tilde{\boldsymbol{b}}
$$

We refer to Eq. (7) as the reduced model. Unfortunately, we do not know a-priori what snapshots to collect, so the algorithm proposed by Pasetto et al. [13] is used iteratively to collect snapshots and build the $\boldsymbol{P}$ matrix. The procedure for constructing $\boldsymbol{P}$ is summarized as follows:

1. Select a hydraulic conductivity combination $(\boldsymbol{k})$, run the full model, collect the snapshots at the times according to the algorithm proposed by Siade et al. [11] and store them in $\chi \subseteq \boldsymbol{X}$, and perform SVD on $\chi$ such that $\chi=\hat{\boldsymbol{P}} \Sigma \boldsymbol{V}^{T}$, where $\hat{\boldsymbol{P}} \subseteq \boldsymbol{P}$

a. If $\boldsymbol{P}$ does not exist, create $\boldsymbol{P}$ from the first principal vector of $\hat{\boldsymbol{P}}$ and construct a reduced model. 
2. Calculate $\|\boldsymbol{e}\|_{2}$

a. While $\|\boldsymbol{e}\|_{2} \geq \tau$, augment $\boldsymbol{P}$ with sequential principal vectors from $\hat{\boldsymbol{P}}$ and construct a new reduced model.

3. Calculate $d\left\|\boldsymbol{r}_{e}\right\|$

4. If $d\left\|\boldsymbol{r}_{e}\right\| \geq \tau$, return to step 1 and select the next $\boldsymbol{k}$ in the parameter space at which to take snapshots by solving the following greedy search [13]:

$$
\underset{\boldsymbol{k}}{\arg \max } d\left\|\boldsymbol{r}_{e}\right\|
$$

Else if $d\left\|\boldsymbol{r}_{e}\right\|<\tau$, output $\boldsymbol{P} \in R^{N_{n} \times n_{p}}$ and exit.

McPhee and Yeh [10] showed that POD could reduce a groundwater model by several orders of magnitude while sustaining a loss in accuracy of less than one percent. Siade et al. [11] demonstrated that a groundwater model containing over 200,000 nodes and thus over 200,000 equations could be reduced to a model with less than 10 equations through the application of POD. The reduced model runs approximately 1,000 times faster than the full model with acceptable error. By replacing the full, computationally expensive model with a reduced, computationally inexpensive model, we are able to feasibly use a GA to search for the optimal network design.

\section{1.d. Experimental Design}

Now we have a set of methods that allow us to feasibly search for the network design that gives us the most useful information. However, we still are faced with two questions: What is useful information? How do we quantify the amount of information gained? Answering these questions 
has been the topic of much research spanning diverse fields of study. Many of them share common elements.

Since the 1920 s statistical methods have been accepted as the primary means to answer these questions. For example, the Jacobian matrix $\left(\boldsymbol{J}_{\boldsymbol{d}}\right)$ is defined as

$$
\boldsymbol{J}_{d}=\left(\begin{array}{ccc}
\frac{\partial o_{1}}{\partial \theta_{1}} & \cdots & \frac{\partial o_{1}}{\partial \theta_{N}} \\
\vdots & \ddots & \vdots \\
\frac{\partial o_{n_{o b s}}}{\partial \theta_{1}} & \cdots & \frac{\partial o_{n_{o b s}}}{\partial \theta_{N}}
\end{array}\right)
$$

where $n_{o b s}$ is the total number of observations taken, which may vary spatially and temporally; $N$ is the total number of parameters of interest; and $\frac{\partial o_{i}}{\partial \theta_{j}}\left(i=1, \ldots, n_{o b s} ; j=1, \ldots, N\right)$ is the sensitivity of the $i^{\text {th }}$ observation $\left(o_{i}\right)$ to changes in the $j^{\text {th }}$ parameter $\left(\theta_{j}\right)$ that contains information, expressed by the sensitivities, about the system of interest. These sensitivities provide useful information, as they show how the system responds to changes in the parameters of interest. Three methods have been proposed over the years to calculate the sensitivities of a system: (1) the parameter perturbation method, (2) the sensitivity equation method, and (3) the adjoint state method [20]. A convenient method to use is the perturbation method described as

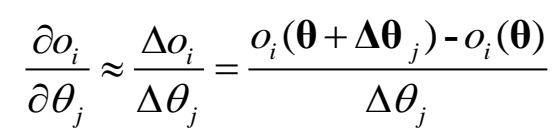

where $\boldsymbol{\theta}$ is a vector of the estimated parameter values, $o_{i}(\boldsymbol{\theta})$ is the $i^{\text {th }}$ model simulated value using the parameter values in $\boldsymbol{\theta}, \Delta \theta_{j}$ is a small increment of the $j^{\text {th }}$ parameter (called the perturbation of $\theta_{j}$ ), and $\Delta \boldsymbol{\theta}_{j}$ is a vector in which all elements are zero except for the $j^{\text {th }}$ element, 
which is equal to $\Delta \theta_{j}$ [21]. Using the parameter perturbation method to estimate sensitivities for general models requires $(N+1)$ model calls (a baseline model solution and $N$ model runs to estimate the sensitivities) [20]. In general, there are two requirements for making accurate estimates of $\frac{\partial o_{i}}{\partial \theta_{j}}$ through the use of Eq. (9). First, $\Delta \boldsymbol{\theta}_{j} \square \boldsymbol{\theta}$ and second, $\boldsymbol{\theta}$ must be close to the true parameter values. As noted, in the real world we cannot take observations at infinitesimally fine spatial and temporal resolutions; consequently, we can think of the experimental design problem as a problem of finding which elements of this theoretically, infinitely large Jacobian matrix should be included in the analysis. This answers the first question (What is useful information?) raised above.

To answer the second question (How do we quantify the amount of information gained?) we adopt the concept of the information matrix (I) from statistics as follows:

$$
\boldsymbol{I}=\boldsymbol{J}_{\boldsymbol{d}}{ }^{T} \times \boldsymbol{J}_{\boldsymbol{d}} \times \boldsymbol{W}
$$

where $\boldsymbol{W}$ is a user-specified weighting matrix. Under the assumptions that 1) a least-squares error criterion is used for parameter estimation; 2) the observation errors are uncorrelated with equal variance; and 3) $\boldsymbol{W}$ is the identity matrix, the information matrix defined in Eq. (10) is equivalent to the inverse of the covariance matrix of the estimated parameters $(\boldsymbol{C}(\boldsymbol{\theta}))[22,23]$. Eq. (11) shows the covariance matrix. This matrix contains the variance of the estimated parameter values $\left(\sigma^{2}\left(\theta_{i}\right)\right)$ and the covariance of estimated parameters to each other $\left(\sigma\left(\theta_{i}, \theta_{j}\right)\right)$. 


$$
\boldsymbol{C}(\boldsymbol{\theta})=\left[\begin{array}{cccc}
\sigma^{2}\left(\theta_{1}\right) & \sigma\left(\theta_{1}, \theta_{2}\right) & \ldots & \sigma\left(\theta_{1}, \theta_{n}\right) \\
\sigma\left(\theta_{2}, \theta_{1}\right) & \sigma^{2}\left(\theta_{2}\right) & \ddots & \vdots \\
\vdots & \ddots & \ddots & \sigma\left(\theta_{n-1}, \theta_{n}\right) \\
\sigma\left(\theta_{n}, \theta_{1}\right) & \cdots & \sigma\left(\theta_{n}, \theta_{n-1}\right) & \sigma^{2}\left(\theta_{n}\right)
\end{array}\right]
$$

We could use any number of methods to quantify the amount of information contained in a given covariance or information matrix. Commonly used methods include measuring the trace (Aoptimality), determinant (D-optimality), or minimum eigenvalue (E-optimality) of the covariance matrix [24]. The most commonly used optimality criterion for groundwater studies is the maximal information criterion $[22,25]$, which measures the trace of the information matrix. Under the assumptions mentioned above, (i.e. the information matrix is equivalent to the inverse of the covariance matrix of the estimated parameters), a design is said to be D-optimal if it maximizes the determinant of the information matrix, while a design is said to be E-optimal if it maximizes the minimum eigenvalue of the information matrix. It can be shown that a design that is A-optimal (minimizes the trace of the covariance matrix) also maximizes the trace of the information matrix and the information received, thus making that design maximal informationoptimal. We may include a set of constraints in the experimental design to constrain the feasible solution space. In the past A, D, and maximal information-optimality have been used to solve experimental design problems in the context of groundwater modeling. A-optimality has been used for unconfined aquifer parameter estimation [26]. D-optimality has been used for designing an optimal multi-objective observation network for parameter estimation and model discrimination [27]; for optimal pumping tests for parameter estimation [28]; and for optimal observation network design for dispersion parameters [29]. Maximal information-optimality has been used for optimal observation networks for confined aquifer parameter estimation [25] and for transport parameter estimation [22]. Cleveland and Yeh [30] pointed out that it is 
advantageous to use maximal information optimality over A- or D-optimality because the maximal information criterion can be computed directly in a straightforward manner without matrix inversion. McCarthy and Yeh [31] performed a study not only to find the optimal design but also evaluate if the amount of information gathered is useful for achieving some objective. Chang et al. [32] studied data sufficiency for an optimal network design.

Since the goal of our experimental design is to gain information about the unknown hydraulic conductivities, the Jacobian matrix is the matrix of sensitivities of drawdown observations to changes in hydraulic conductivity. Thus,

$$
\boldsymbol{J}_{d}=\left(\begin{array}{ccc}
\frac{\partial s_{1}}{\partial K_{1}} & \cdots & \frac{\partial s_{1}}{\partial K_{N}} \\
\vdots & \ddots & \vdots \\
\frac{\partial s_{n_{o b s}}}{\partial K_{1}} & \cdots & \frac{\partial s_{n_{o b s}}}{\partial K_{N}}
\end{array}\right)
$$

where the elements in the Jacobian matrix $\left(\frac{\partial s_{i}}{\partial K_{j}}\right)$ are approximated:

$$
\frac{\partial s_{i}}{\partial K_{j}} \approx \frac{s_{i}\left(\boldsymbol{k}+\Delta \boldsymbol{k}_{j}\right)-s(\boldsymbol{k})}{\Delta K_{j}}
$$

where $s_{i}(\boldsymbol{k})$ is the $i^{\text {th }}$ model simulated observation (drawdown) using the estimated hydraulic conductivities in $\boldsymbol{k} \in R^{n_{z}}, \Delta K_{j}$ is the perturbation of the $j^{\text {th }}$ hydraulic conductivity, $\Delta \boldsymbol{k}_{j} \in R^{n_{z}}$ is a vector perturbing the $j^{\text {th }}$ hydraulic conductivity, and $\frac{\partial s_{i}}{\partial K_{j}}$ is the sensitivity of the $i^{\text {th }}$ drawdown to a change in the $j^{\text {th }}$ hydraulic conductivity. As noted before, to make accurate approximations of $\boldsymbol{J}_{d}, \Delta \boldsymbol{k}_{j} \square \boldsymbol{k}$ and $\boldsymbol{k}\left(K_{1}, \ldots, K_{N}\right)$ must be close to the true parameter values. The first 
requirement is fairly simple to satisfy. However, meeting the second requires some a-priori knowledge, which may not be available. If we cannot acquire this knowledge a-priori we could acquire it through a sequential experimental design [25]. Unfortunately, this method can be too costly and time consuming to be feasible, particularly in the context of groundwater. We also could place an a-priori probability distribution on the parameters to derive an a-posteriori information matrix [33]. A third option would be to perform the optimization using the metaJacobian matrix as opposed to the standard Jacobian matrix [34]. The standard Jacobian matrix contains sensitivities of observations to changes in hydraulic conductivity at a set value of hydraulic conductivity. On the other hand, we form the meta-Jacobian matrix by taking a number of standard Jacobians matrices formed at various values of hydraulic conductivities and augmenting all the matrices together. This optimization would give us a design that would be potentially sub-optimal for all values of hydraulic conductivity but would provide a near-optimal amount of information across the widest range of values. Unfortunately, this design cannot set a lower limit on the amount of information obtained in the worst-case scenario.

\section{Methods}

\section{2.a. Experimental Design}

We choose maximal information-optimality (maximizing the trace of $\boldsymbol{I}$ ) as the fitness criterion for this optimization study. The optimization employs discrete decision variables whose range spans the nodes of the finite element mesh used to model the aquifer. Thus the observation locations chosen will fall on the nodes of that mesh. Furthermore, we define discrete pumping and observation zones in the aquifer. These zones define areas of the aquifer in which observations wells or pumping wells may be placed. These two zonation types are physically 
related to each other as they cannot overlap but are distinct from the aquifer's hydrologic zones that define areas of uniform hydrologic properties (e.g. hydraulic conductivity, specific storage, depth, etc.). The constraints applied to this optimization are as follows: (1) at most one observation well may be placed in an observation zone; and (2) the total number of observation wells cannot exceed some upper limit. This yields the experimental design formulation

$$
\begin{gathered}
\max _{\boldsymbol{\omega} \subset \boldsymbol{\Omega}} \operatorname{Tr}\left(\boldsymbol{J}_{d}{ }^{T} \times \boldsymbol{J}_{d} \times \boldsymbol{W}\right) \\
\text { s.t. } \sum_{i=\text { zone } j} x_{i} \leq 1 \forall j \\
\sum_{i=1}^{N_{n}} x_{i} \leq n_{\text {obswell }}
\end{gathered}
$$

where $n_{\text {obswell }}$ is the total number of allowable observation wells, zone $j$ indicates all the nodes in the $j^{\text {th }}$ observation zone, $N_{n}$ is the total number of nodes in the model, $\omega \in R^{n_{\text {obswell }}}$ is some feasible set of observation well locations, $\boldsymbol{\Omega}$ is the space of all feasible sets of observation well locations, and $\boldsymbol{x} \in R^{N_{n}}$ contains binary variables (equal to 1 if an observation is taken at that node and 0 otherwise). In this study we choose $\boldsymbol{W}$ to be the identity matrix of size $n_{z}$, where $n_{z}$ is the total number of hydrologic zones with unknown hydraulic conductivity. Rather than depending on acquiring some a-priori knowledge, performing a sequential design, or employing a meta-Jacobian, we choose to perform a robust experimental design that can be described by the following max-min formulation [35]:

$$
\begin{gathered}
\max _{\boldsymbol{\omega} \subset \boldsymbol{\Omega}}\left(\min _{\boldsymbol{k} \subset \hat{\boldsymbol{K}}} \operatorname{Tr}\left(\boldsymbol{J}_{d}{ }^{T} \times \boldsymbol{J}_{d} \times \boldsymbol{W}\right)\right) \\
\text { s.t. } \sum_{i=\text { zone } j} x_{i} \leq 1 \forall j \\
\sum_{i=1}^{N_{n}} x_{i} \leq n_{\text {obswell }}
\end{gathered}
$$


where $\hat{\boldsymbol{K}}$ is the space of feasible hydraulic conductivities. In other words, the robust design is the one that provides the maximum amount of information in the worst-case scenario. We assume that the zonation pattern of the aquifer already has been determined from prior geological information. To solve this optimization problem, we first discretize the zonal hydraulic conductivity into a number of increments. Specifically, for each of the $n_{z}$ hydrologic zones we discretize the feasible range for its hydraulic conductivity into $n_{l}$ increments. We then form all possible combinations of hydraulic conductivity in all hydrologic zones, resulting in a total of $n_{l}^{n_{z}}$ combinations. We will refer to this set of combinations as $K \in R^{n_{z} \times n_{l}^{n_{z}}}$ and solve the optimization problem over this set. In the robust experimental design, minimization is obtained by the proper choice of $\boldsymbol{k} \subset \boldsymbol{K}$. This yields the following robust experimental design formulation:

$$
\begin{gathered}
\max _{\boldsymbol{\omega} \subset \boldsymbol{\Omega}}\left(\min _{\boldsymbol{k} \subset \hat{\boldsymbol{K}}} \operatorname{Tr}\left(\boldsymbol{J}_{d}{ }^{T} \times \boldsymbol{J}_{d} \times \boldsymbol{W}\right)\right) \\
\text { s.t. } \sum_{i=\text { zone } j} x_{i} \leq 1 \forall j \\
\sum_{i=1}^{N_{n}} x_{i} \leq n_{\text {obswell }}
\end{gathered}
$$

Note that Eq. (15) optimizes over the full, continuous hydraulic conductivity space while Eq. (16) optimizes over a discretized hydraulic conductivity space. This max-min combinatorial optimization is nonlinear, non-convex, and extremely difficult to solve. To solve this problem, we use the following systematic search procedure:

1. Discretize $\hat{\boldsymbol{K}}$ into $n_{l}^{n_{z}}$ parameter combinations (i.e. combinations of zonal hydraulic conductivities) to produce $\boldsymbol{K} \in R^{n_{z} \times n_{l}^{n_{z}}}$. 
2. For each combination, use Eq. (13) to approximate the elements of the Jacobian matrix; solve Eq. (14), which searches for some observation network $(\boldsymbol{\omega})$ that is an optimal observation network $\left(\tilde{\boldsymbol{\omega}}_{i}\right)$ for the given parameter combination; and perform an exhaustive search over the remaining $n_{l}^{n_{z}}-1$ parameter combinations to identify the worst-case scenario (i.e. the combination for which $\tilde{\boldsymbol{\omega}}_{i}$ provides the least amount of information). The score (i.e. the value of the objective function for a particular $\tilde{\boldsymbol{\omega}}_{i}$ ) associated with the worst-case scenario is referred to as the robust score.

3. After completing step 2 for all parameter combinations, choose the robust design by finding the $\tilde{\boldsymbol{\omega}}_{i}$ that has the maximum worst-case score.

In addition to finding the robust experimental design, we also find what we term the High Frequency Observation Well (HFO) design, described as follows. If we let $\tilde{\boldsymbol{\omega}}_{i}^{(j)}$ be the $j^{\text {th }}$ optimal location in $\tilde{\boldsymbol{\omega}}_{i}$ and $\hat{\boldsymbol{\omega}}$ be the set of all $\tilde{\boldsymbol{\omega}}_{i}$ such that

$$
\hat{\boldsymbol{\omega}}=\bigcup_{i=1}^{n_{l}^{n_{z}}} \tilde{\boldsymbol{\omega}}_{i}
$$

then the HFO is the $n_{\text {obswell }} \tilde{\boldsymbol{\omega}}_{i}^{(j)} \subset \hat{\boldsymbol{\omega}}$ that occurs with the highest frequency. In other words, we take the set of all $\tilde{\boldsymbol{\omega}}$ 's and find the observation locations that occur most frequently in that set. We then create a contour plot of the frequency (scaled to sum to 1) with which some $\tilde{\boldsymbol{\omega}}_{i}^{(j)}$ occurs in $\hat{\boldsymbol{\omega}}$. This gives us a map that estimates the probability distribution of optimal locations. In a way this is similar to a Monte Carlo approach to estimating the probability of a location residing in the optimal set $\left(\omega^{*}\right)$, assuming a uniform prior of hydraulic conductivity where $\omega^{*}$ is the optimal solution to a specific experimental design given the true aquifer parameters. Doing 
this allows us to take a probabilistic rather than deterministic approach to placing observation wells. This approach could have some real-world advantages. For instance, if it is found after the optimization is completed that one of the optimal locations cannot be used and the optimization cannot be re-run, the determination of which feasible location should be used as a replacement can be made based on the plot of the estimated probability distribution.

\section{2.b. Genetic Algorithm}

Wall [36] developed a base GA code (GAlib) that supports many features that lend themselves to solving the particular problem at hand. These features include support for real number genomes, adaptability, and options that allow the implementation of various "flavors" of GA. From this base code, we developed a GA that searches the feasible region by using real number genomes. To do this, we index all possible observation well locations in the full model space (this index is referred to as $\boldsymbol{\Omega}$ ) and the GA creates populations comprised of individuals that represent feasible combinations of observation well locations $(\boldsymbol{\omega} \subset \boldsymbol{\Omega})$. For each individual in a population, the

GA calls the reduced model to approximate the Jacobian $\left(\boldsymbol{J}_{d}\right)$ and Information $(\boldsymbol{I})$ matrices (Eq. (12) and Eq. (10), respectively) to evaluate the individual's fitness (i.e. how well an individual optimizes the objective function). To calculate Eq. (13) in the reduced space, the GA passes $\boldsymbol{M P}$ to the reduced model, where $\boldsymbol{M} \in R^{n_{\text {obswell }}} x N_{n}$ is an observation matrix in which the $i^{\text {th }}$ row vector contains a 1 in the column associated with the $i^{\text {th }}$ element in $\boldsymbol{\omega}$ and 0 s elsewhere. The reduced model calculates the reduced solution $(\boldsymbol{R}(\boldsymbol{k}))$ at the observation times and solves. Eq. (13) in the reduced space through the following equation:

$$
J_{d_{i, j}}=\frac{\partial s_{i}}{\partial K_{j}} \approx \frac{\left(\tilde{\boldsymbol{P}} \boldsymbol{R}\left(\boldsymbol{k}+\Delta \boldsymbol{k}_{j}\right)-\tilde{\boldsymbol{P}} \boldsymbol{R}(\boldsymbol{k})\right)_{i, j}}{\Delta K_{j}}
$$


By calculating the fitness of each individual (i.e. how close the solution is to the solution of Eq. (14)) in the reduced space, we achieve a drastic reduction in computation time.

After evaluating the fitness of the individuals in a population, the GA uses mutations and crossovers to solve the optimization problem of Eq. (14). The GA remembers the elite score (i.e. the best score achieved across all generations) and declares convergence when there is no deviation between the elite score and the best current generation score for 300 generations (i.e. convergence of solution). This process then is repeated exhaustively for all $\boldsymbol{k} \subset \boldsymbol{K}$ to solve the robust experimental design problem (Eq. (16)). Ushijima and Yeh [4] showed that for a fixed $\boldsymbol{k}$, it is possible to solve the experimental design problem of selecting optimal observation locations using a GA that calls a reduced model to estimate the Jacobian matrix. A flowchart of the GA with the POD reduced model appears in Figure 1. 


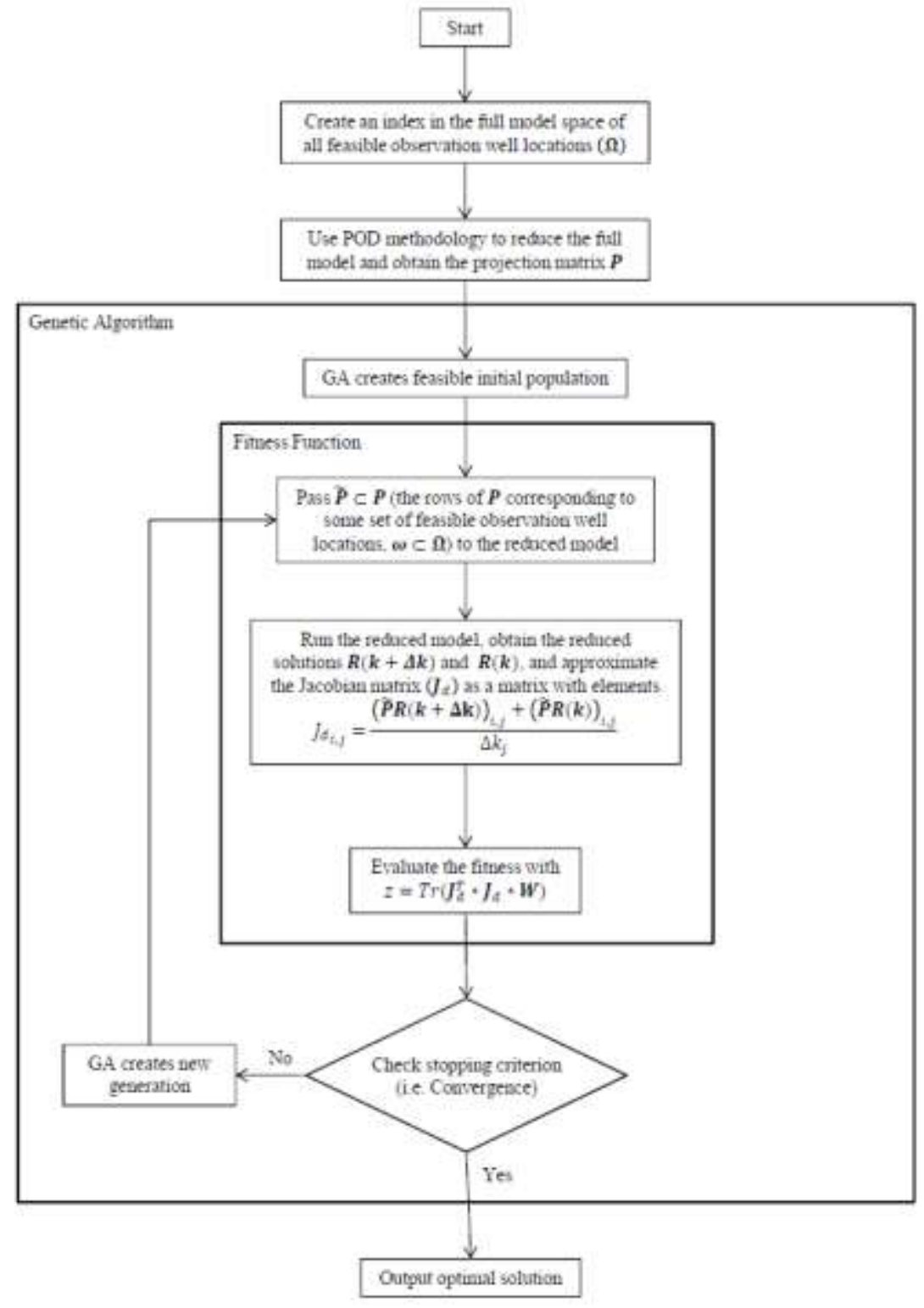

Figure 1. Flowchart of GA

\section{Test Cases}

\section{3.a. One-Dimensional Test Case}

We develop a synthetic, one-dimensional test cast to validate our algorithm (see Figure 2). The setup is similar to ones developed for previous studies related to POD reduced groundwater models $[2,11]$. The aquifer is $100 \mathrm{~m}$ long with a depth and width of $1 \mathrm{~m}$. The aquifer is discretized into cubic cells $1 \mathrm{~m}$ per side. The specific storage $\left(S_{s}\right)$ is $1 \mathrm{~m}^{-1}$ throughout the aquifer (we use a 
large value of $S_{s}$ here to speed-up the solution time to reach a steady state). Two wells pumping at the same rate $\left(1 \mathrm{~m}^{3} /\right.$ day $)$ are placed in the aquifer at node 31 and node 69 and the aquifer is divided into two hydrologic zones with unknown hydraulic conductivity (designated "Unknown Zone 1" and "Unknown Zone 2"), as seen in Figure 2.

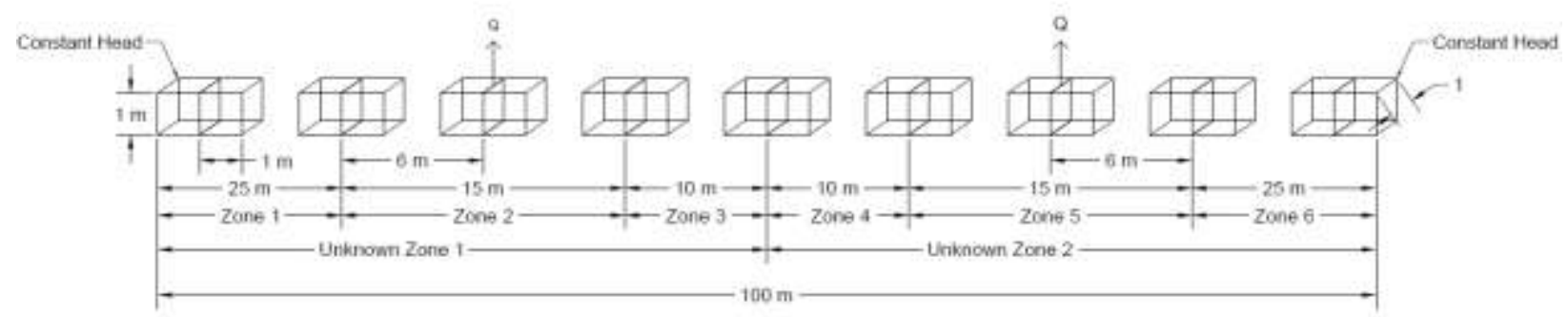

Figure 2. 1-D test case

The upper and lower bounds on hydraulic conductivity throughout the aquifer are $20 \mathrm{~m} /$ day and $0.1 \mathrm{~m} /$ day, respectively. When modeled in SAT2D [37] the full model consists of 303 nodes (as a result of the finite element mesh used to model the aquifer). We reduce this full model to a parameter-independent reduced model containing only 25 equations. On an Intel Core $2.40 \mathrm{GHz}$ i5 CPU the full model takes 14 seconds to complete a run, while the reduced model takes 0.004 seconds to complete a run.

For the experimental design problem, we divide the aquifer into two pumping zones and four observation zones as shown in Figure 2. Zones 2 and 5 are designated as pumping zones while Zones 1, 3, 4, and 6 are designated as observation zones. We discretize the hydraulic conductivity space into three increments $(0.1 \mathrm{~m} /$ day, $10.05 \mathrm{~m} /$ day , and $20 \mathrm{~m} /$ day $)$, yielding $2^{3}=9$ combinations of hydraulic conductivities (corresponding to nine different $\tilde{\boldsymbol{\omega}}_{i}$ 's). The optimal sampling frequency can be determined through experimental design. However, for this study we 
take observations from the beginning to the end of the experiments. We let $\Delta k_{j}=0.01 k_{j} \forall j$ and observations are taken at $0.5,1.5,3,5,9,15,25,40,60$, and 100 days. We investigate four experimental designs designated One, Two, Three, and Four observation well networks, respectively. Table 1 shows the number of model calls required for the algorithm to achieve convergence and the observation well locations chosen for the robust and HFO designs in the algorithm. In addition, Table 1 lists the observation well locations for a balanced design, i.e. a possible a-priori best guess. After finding the robust, HFO, and balanced designs we compare the results in three ways: the robust score (maximum-minimum score), mean score, and maximum score as shown in Figure 3.

Table 1. Number of model calls and observation well locations for the robust, HFO, and balanced designs for the 1-D Test Case

\begin{tabular}{|cccccccc|}
\hline \multicolumn{3}{|c}{ 1 observation well network } & \multicolumn{4}{c|}{ observation well network } \\
\hline Model calls & HFO & Robust & Balanced & Model calls & HFO & Robust & Balanced \\
\hline 141,210 & 75 & 39 & 50 & 201,258 & 39 & 75 & 75 \\
\hline \multicolumn{8}{|c}{} \\
\hline
\end{tabular}




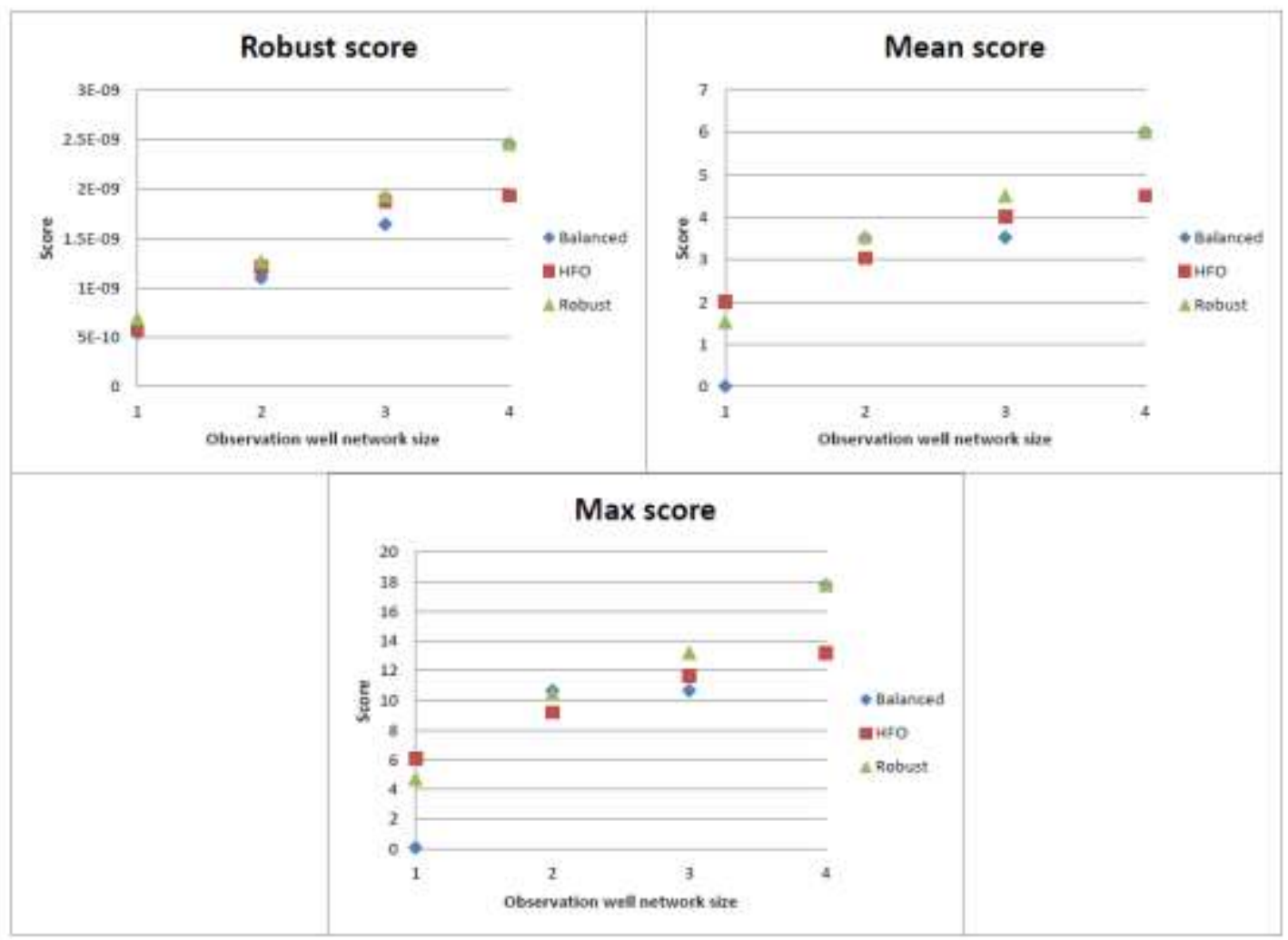

Figure 3. Scores from the 1-D test case

These metrics provide insight into which of the designs would be expected to perform better in the worst case, best case, and on average. It should be noted that the results are very closely tied to the specific experiment setup. In the setup here, as we see, the scores for each of the three metrics are fairly close. As expected by the definition, the robust design outperforms the other designs in robustness. However, in mean score and max score the robust design is sometimes outperformed by the other designs. The balanced design approximates the scores of the optimized designs fairly well but never attains the highest score.

After obtaining the optimal designs, we investigate the inaccuracy introduced to the optimal design by replacing the full model with the reduced model in this $1-\mathrm{D}$ test case. We test the 
robustness of the information from the reduced model by systematically increasing the error tolerance level $(\tau)$ used during reduced model construction. This produces less accurate reduced models; that is, the reduced models with fewer principal vectors. We found that with a oneprincipal vector reduced model, the optimized robust and HFO design locations are very close to the locations selected by the 25-principal vector reduced model. In addition, when using a twoprincipal vector reduced model, the optimized designs select the same locations as the 25principal vector reduced model. This makes sense, as the first few principal vectors capture the dominating characteristics of the full model. For this 1-D case, it appears that the design is not sensitive to the changes in $\tau$. However, information is lost as the number of retained principal vectors is decreased. Figure 4 shows the trace of the information matrix obtained by the robust and HFO designs as well as the reduced model error (RMSE) as a function of the number of retained principal vectors. 


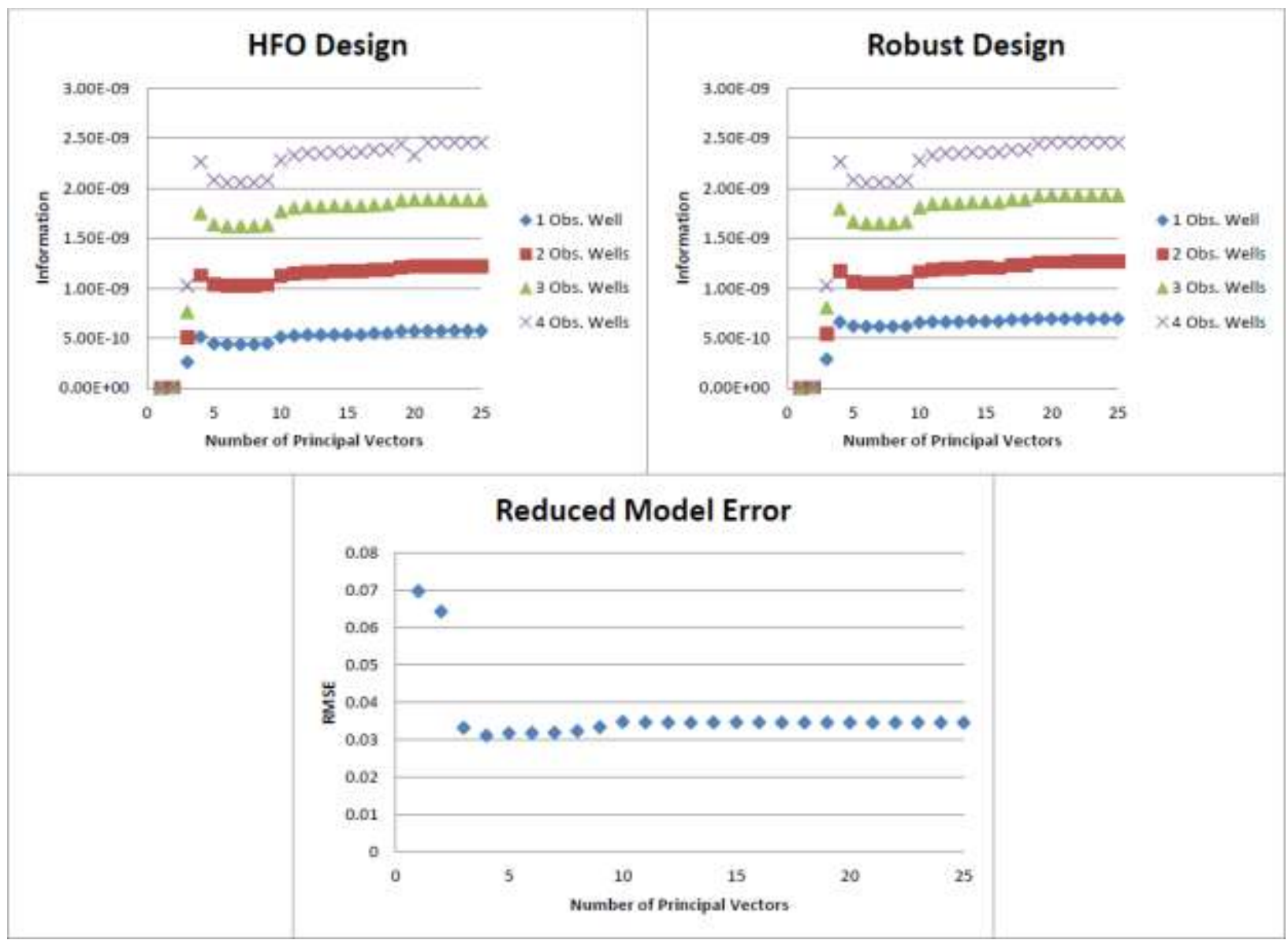

Figure 4. Information vs. number of retained principal vectors

As expected, the amount of information obtained by the HFO and robust designs both increase as a function of increasing number of retained principal vectors. The outliers to this trend are artifacts of the model reduction. It appears that the information loss plateaus around 10 principal vectors. This implies that there is virtually no information lost by reducing the number of principal vectors from 25 to 10 , though this is highly model-dependent. 

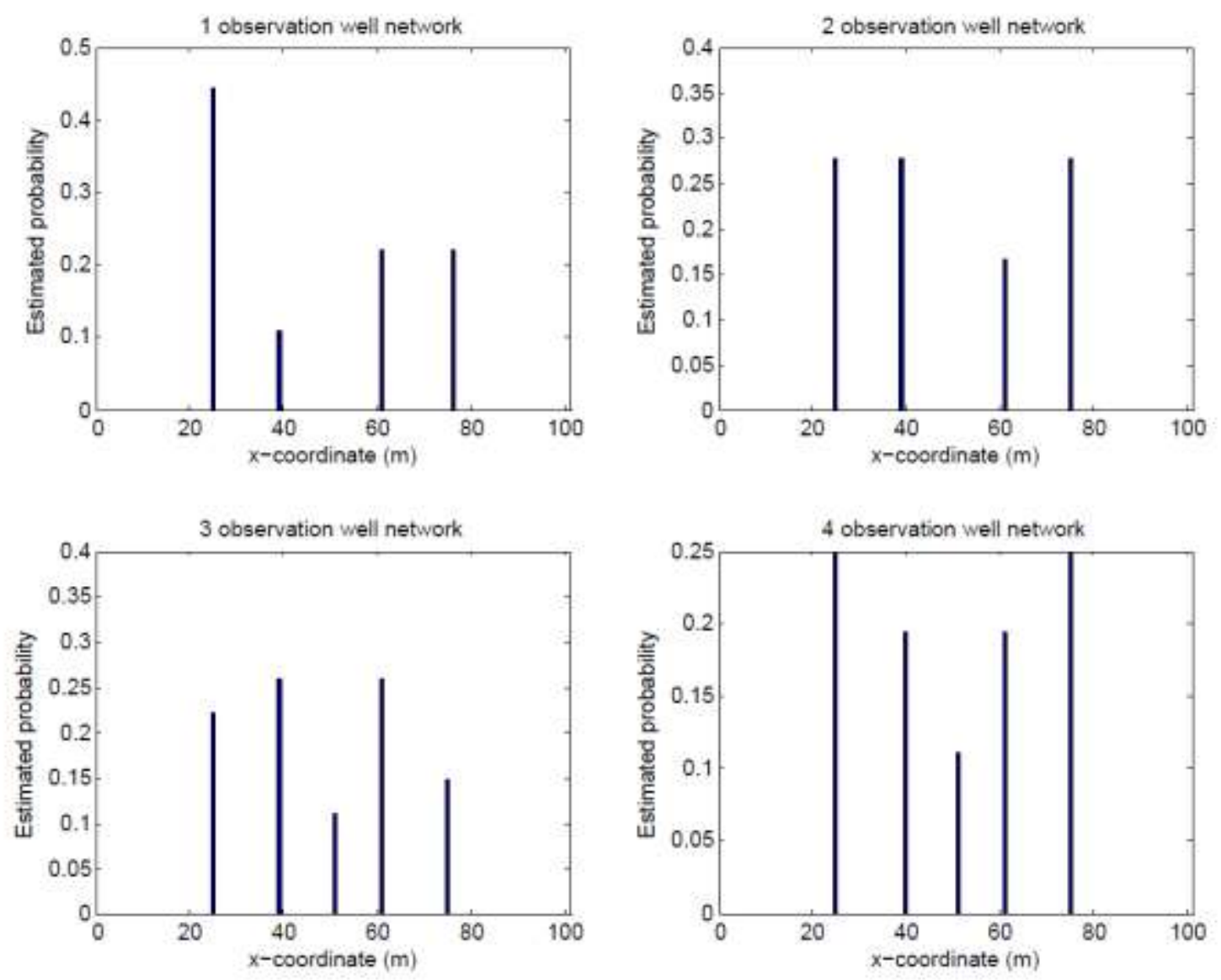

Figure 5. Estimated probability for a location to be an optimal location (1-D test case) Figure 5 shows an approximation of the probability of a location serving as an optimal location (i.e. being a part of $\left.\omega^{*}\right)$. We achieve this by taking the frequency at which a location is an optimal location and normalizing the frequencies for a particular experimental design so that the frequencies sum to one. From this figure we see that, given the specific experimental setup, the observation wells tend to be located as close to the pumping wells as possible with a fairly balanced distribution around each pumping well. The results of the optimization are consistent with expected results. We deduce the expected results by considering that the optimality criteria chosen will cause the algorithm to choose nodes in the observation zones that are as close as 
possible to the pumping wells. Because of this, the nodes near the center of the aquifer will not be chosen until the algorithm is forced to include them because of the constraints placed on the optimization, particularly the constraint that no more than one observation well can be placed in any observation zone. Based on the hydraulic conductivity variation used, we would expect a balanced distribution around each pumping well. Imbalances in estimated probabilities for the expected closest locations are the result of random noise as the algorithm chooses between equally likely locations. A larger sample size would conceal this random noise.

\section{3.b. Two-Dimensional Test Case}

We construct a 2-D horizontal test case to assess the algorithm on a large-scale, real-world-sized model. The model simulates the groundwater flow in a confined aquifer of the Oristano plain in west-central Sardinia, Italy [12,38]. We assume the aquifer is surrounded on all sides by Dirichlet boundaries and is divided into seven hydrologic zones with unknown hydraulic conductivities, as seen in Figure 5.

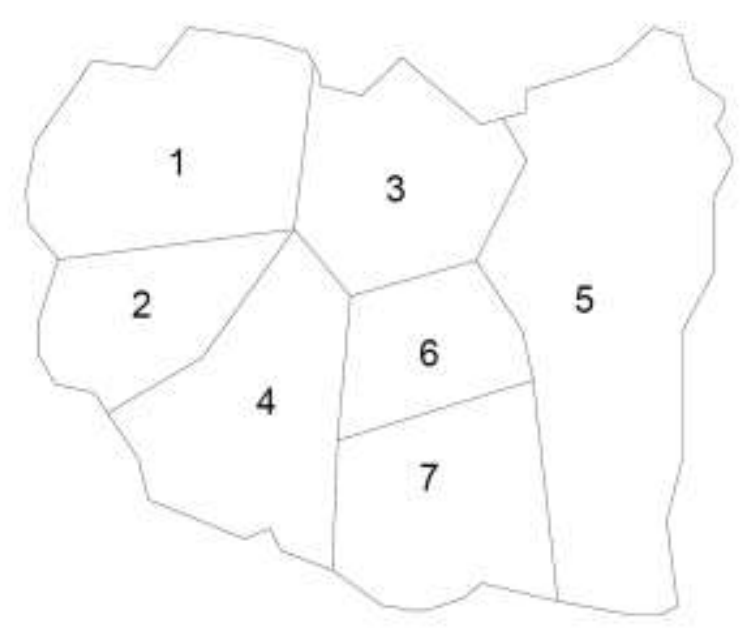

Figure 6. 2-D test case hydrologic zones

The assumed properties of the hydrologic zones appear in Table 2. 
Table 2. 2-D test case known hydrologic parameters

\begin{tabular}{|ccc|}
\hline Zone & $\mathrm{S}_{\mathrm{s}}(1 / \mathrm{m})$ & Depth $(\mathrm{m})$ \\
\hline 1 & $1.20 \mathrm{E}-05$ & 100 \\
\hline 2 & $2.70 \mathrm{E}-05$ & 85 \\
\hline 3 & $1.00 \mathrm{E}-05$ & 110 \\
\hline 4 & $1.70 \mathrm{E}-05$ & 100 \\
\hline 5 & $2.10 \mathrm{E}-05$ & 96 \\
\hline 6 & $3.40 \mathrm{E}-05$ & 105 \\
\hline 7 & $1.00 \mathrm{E}-05$ & 104 \\
\hline
\end{tabular}

Twenty pumping wells are scattered throughout the aquifer (see Figure 8), all extracting water from the aquifer at the same rate $(1,000 m /$ day $)$. Using SAT2D [37], the full model contains 29,197 nodes and 57,888 elements. Using the POD methodology described earlier, we construct a parameter-independent reduced model that contains only 113 equations. There is always some error associated with model reduction. To verify that the error for the reduced model is acceptable we randomly select 500 hydraulic conductivity combinations from a uniform distribution $\sim U(0.1 \mathrm{~m} / d a y, 20 \mathrm{~m} / d a y)$, run the full and reduced models for each combination, and compare the results. For each combination, we calculate and sort the norm of the error and the norm of the relative error between the full and reduced models at steady state in descending order based on the norm of the error. Figures 6 and 7 show the error exceedance curves between the full and reduced models. These figures are similar to breakthrough curves and can be used to see how many parameter combinations have a worse error than some specified cutoff. For example, Figure 6 shows that the maximum norm of the error at steady state for any parameter combination is 0.004 and the norm of the error is greater than 0.001 (the error tolerance used in the algorithm to construct the reduced model) for only 20 of the 500 parameter combinations sampled. Figure 7 shows the relative error (i.e. the norm of the error divided by the magnitude of the drawdown); we can see that the error introduced by model reduction is insignificant for all 500 combinations. 


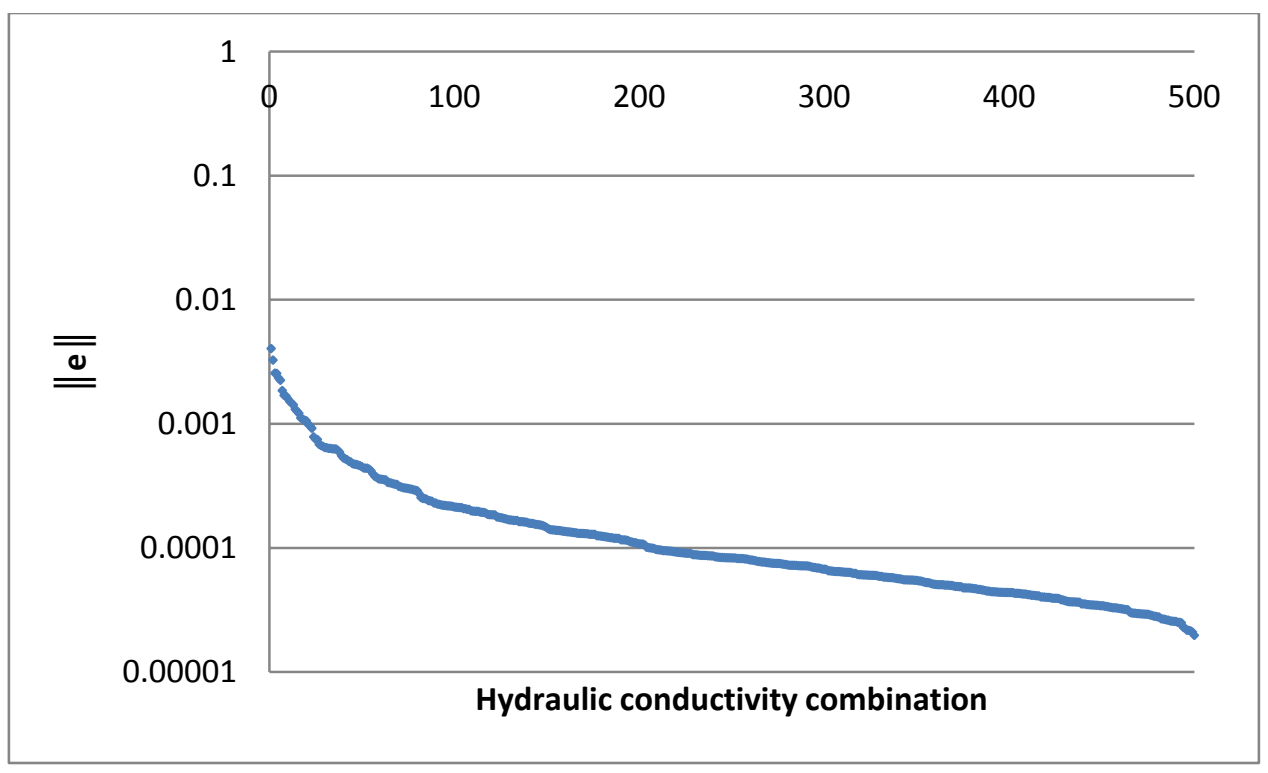

Figure 7. Norm of the error between the full and reduced model results at steady state

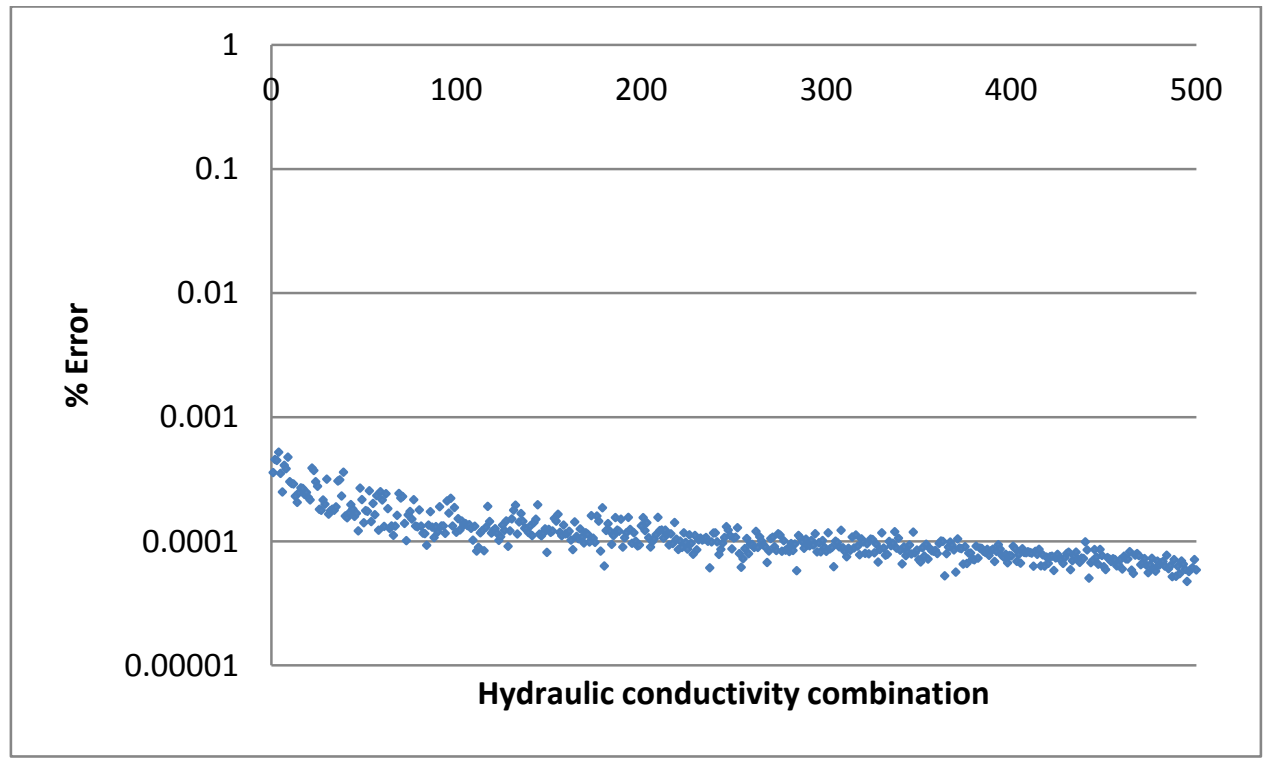

Figure 8. Percent error between the full and reduced model results at steady state When run on an Intel Core $2.40 \mathrm{GHz}$ i5 CPU, the full model takes 64 seconds to complete a run while the reduced model takes only 0.027 seconds to complete a run.

For the experimental design problem we divide the aquifer into 22 arbitrary pumping/observation zones. Pumping wells exist in Zones 3, 5, 6, 7, 9, 10, 11, 13, 14, and 15. These zones are 
designated as pumping zones and the rest (Zones 1, 2, 4, 8, 12, and 16 through 22) are designated as observation zones. For practicality, we overlay a coarse grid on the aquifer with a spacing of $500 \mathrm{~m}$ and only allow observation wells to be placed on the grid nodes that fall within an observation zone. After performing this, we find 328 feasible observation well locations. Sun and Yeh [35] demonstrated that considering only the upper and lower bounds of the hydraulic conductivity will provide sufficient information to develop a robust experimental design. Therefore, when solving the max-min experimental design problem, we only consider the upper and lower bounds of the hydraulic conductivity $(0.1 \mathrm{~m} /$ day and $20 \mathrm{~m} /$ day $)$, yielding a total of $2^{7}=128$ hydraulic conductivity combinations. The number of feasible observation well locations and hydraulic conductivity combinations define the dimension of the experimental design problem. The experimental design problem setup appears in Figure 8.

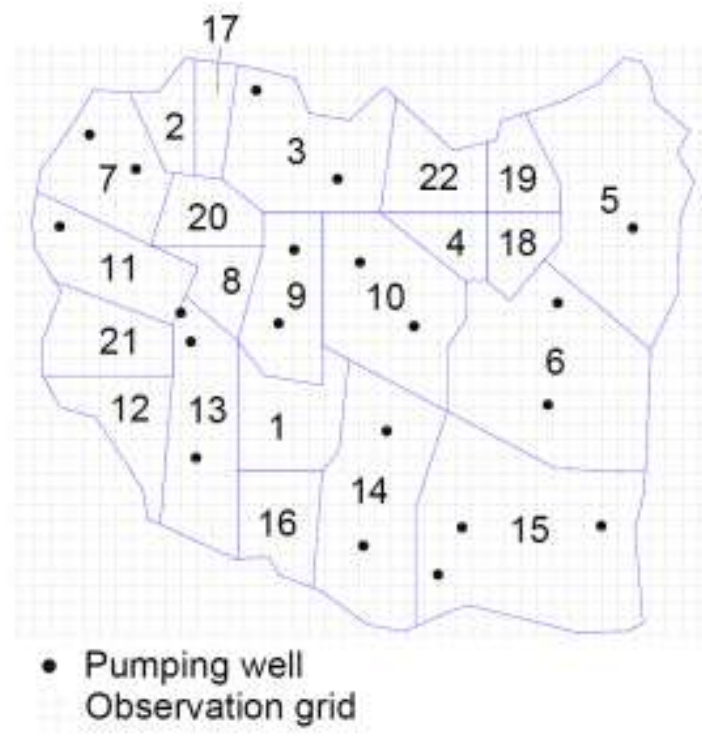

Figure 9. 2-D test case experimental setup

We let $\Delta k_{j}=0.01 k_{j} \forall j$, take observations at $0.1,0.5,1,5,10$, and 50 days and investigate a number of experimental designs. First, we optimize a single observation well network, then we 
optimize a two-observation well network, and so on until finally we optimize a 12-observation well network. The results are shown in Figures 9-15.
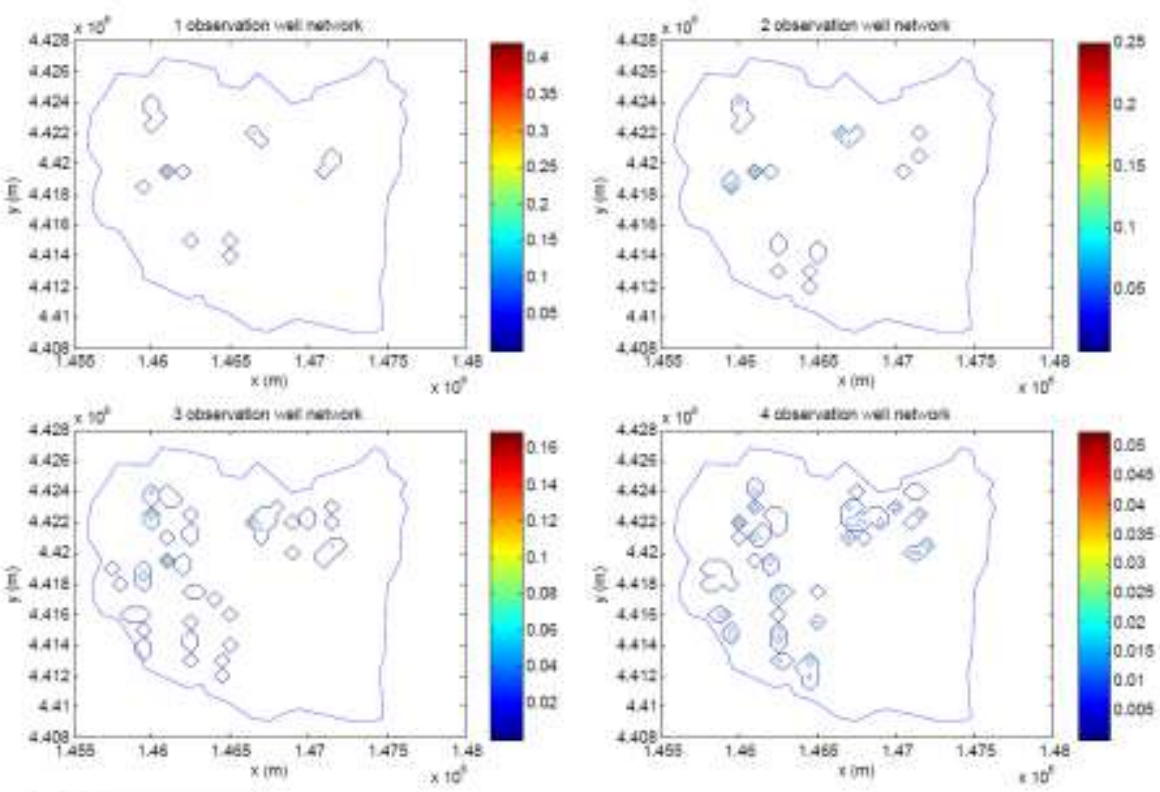

(9) Estmad prosusaty

Figure 10. Estimated probability for a location to be an optimal location for the 1, 2, 3, and 4 observation well networks (2-D Test Case) 

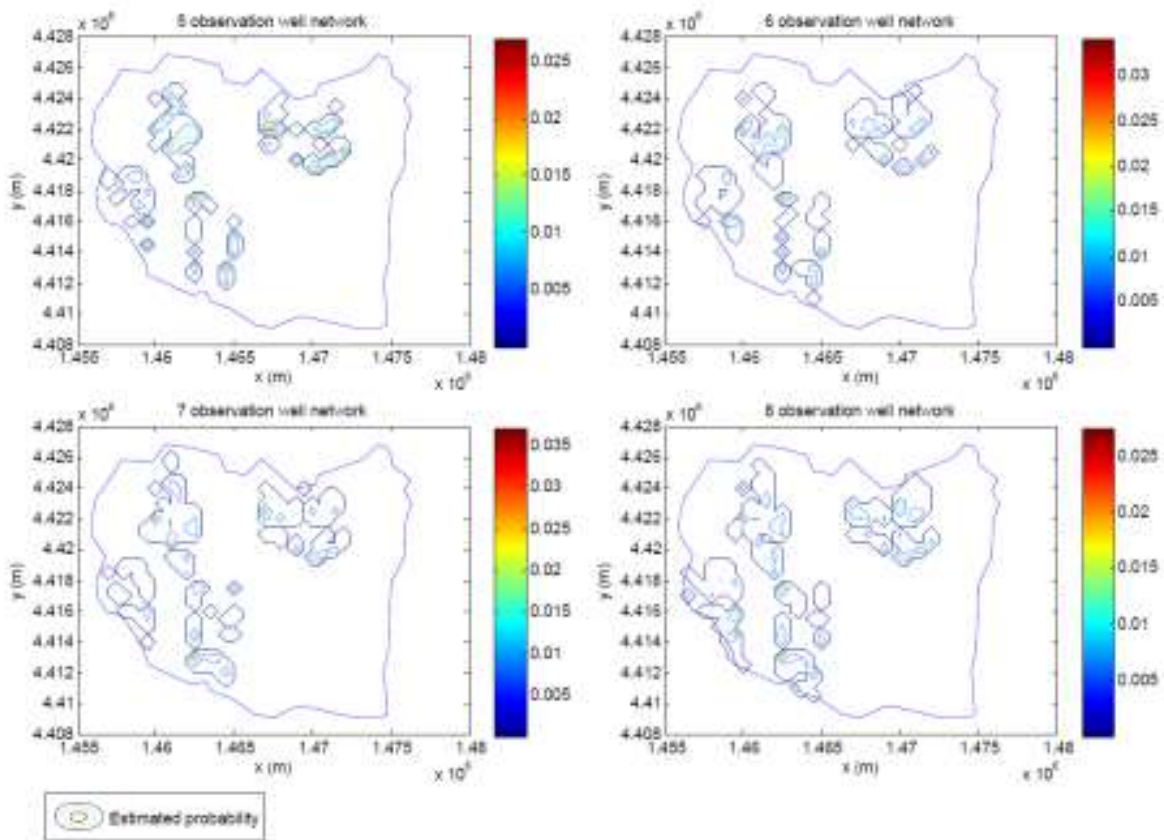

Figure 11. Estimated probability for a location to be an optimal location for the 5, 6, 7, and 8 observation well networks (2-D Test Case)
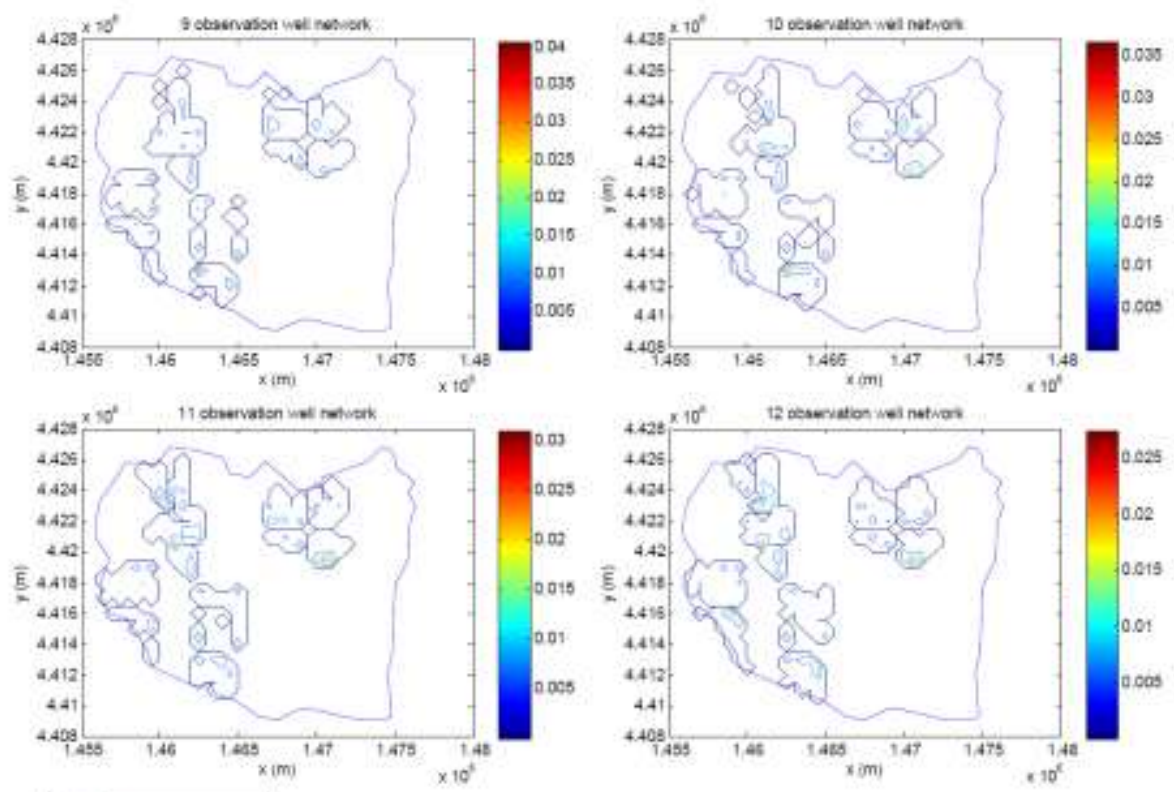

(3) Intrated protacity

Figure 12. Estimated probability for a location to be an optimal location for the $9,10,11$, and 12 observation well networks (2-D Test Case) 

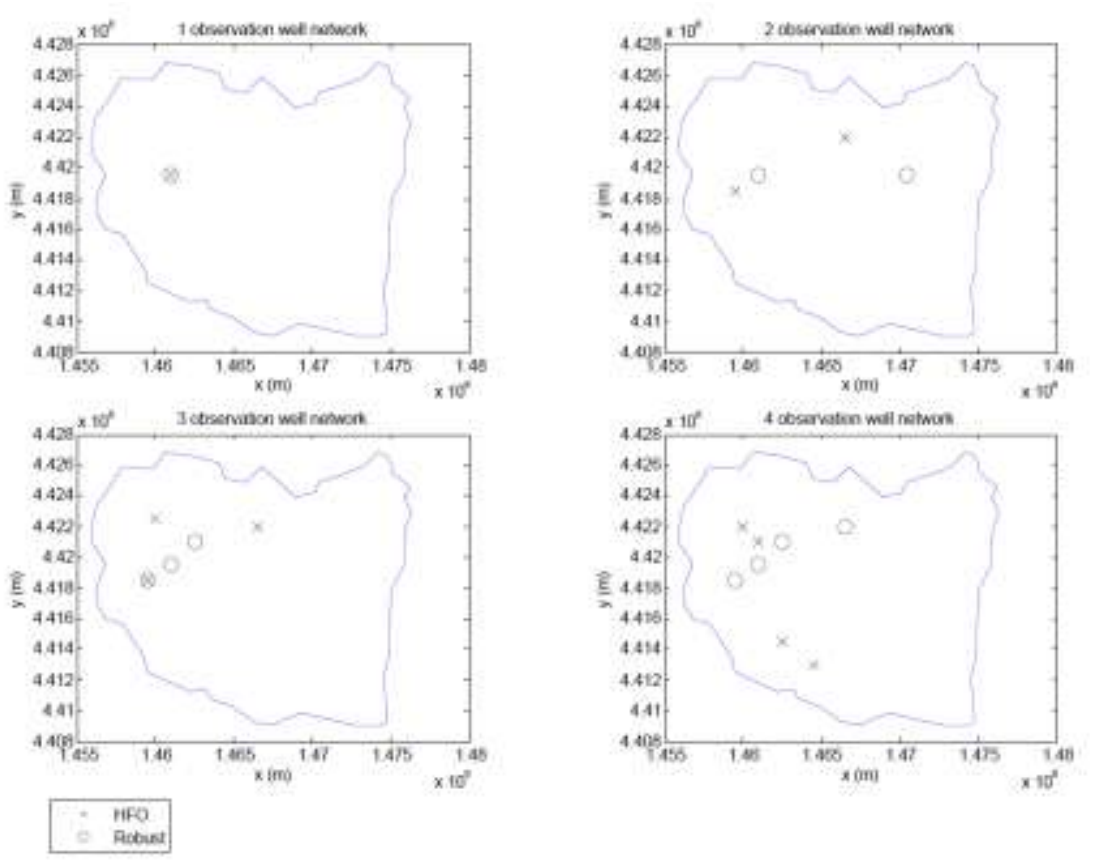

Figure 13. Robust and HFO designs for 1, 2, 3, and 4 observation well networks (2-D Test Case)
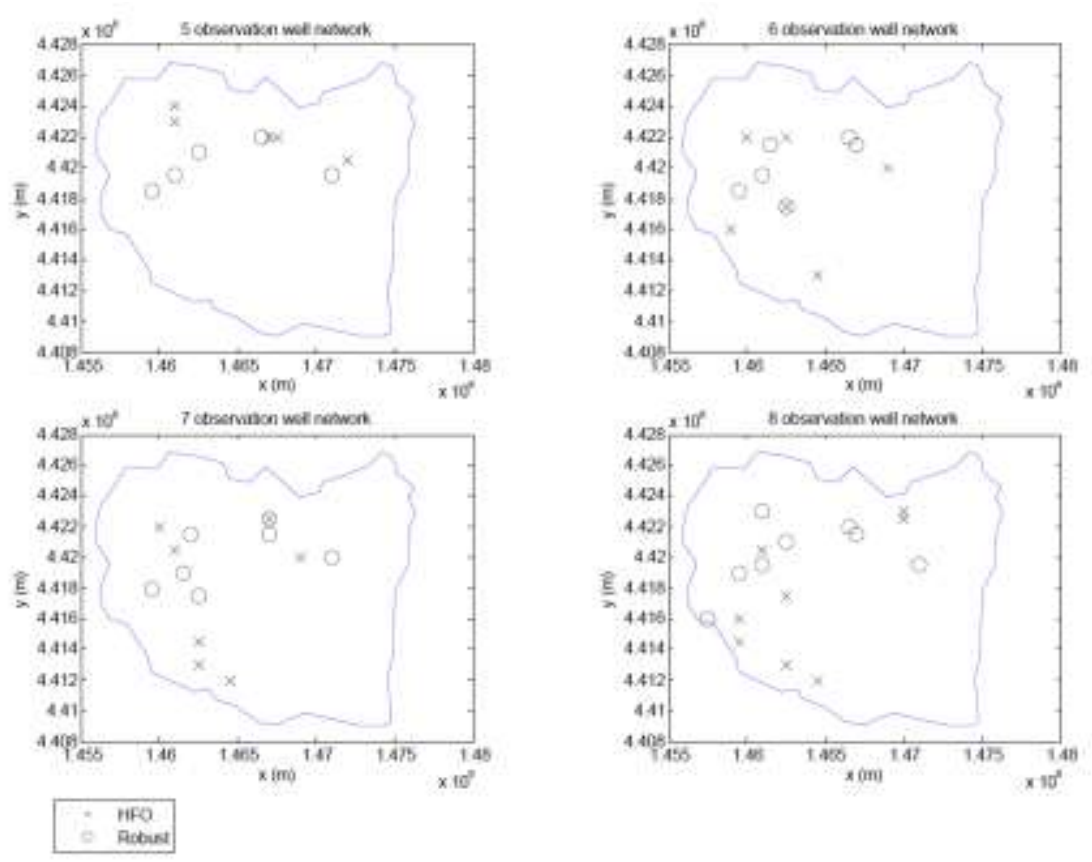

Figure 14. Robust and HFO designs for 5, 6, 7, and 8 observation well networks (2-D Test Case) 

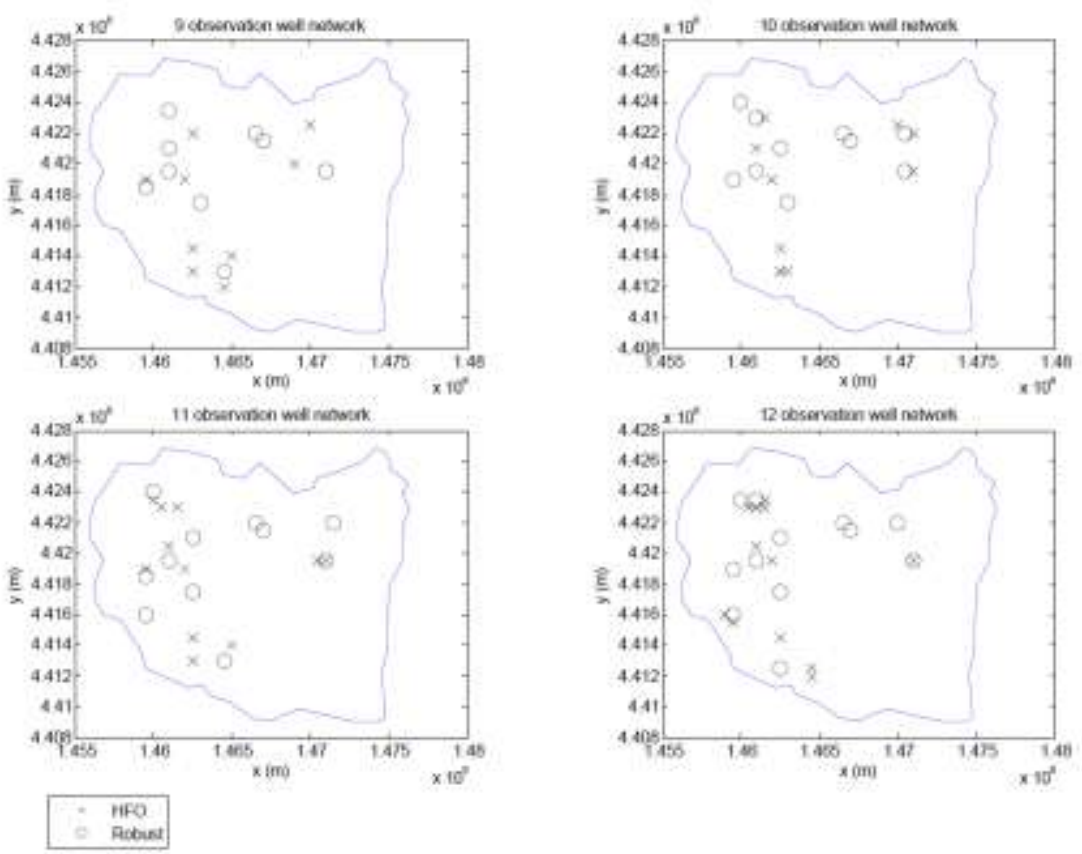

Figure 15. Robust and HFO designs for 9, 10, 11, and 12 observation well networks (2-D Test Case)

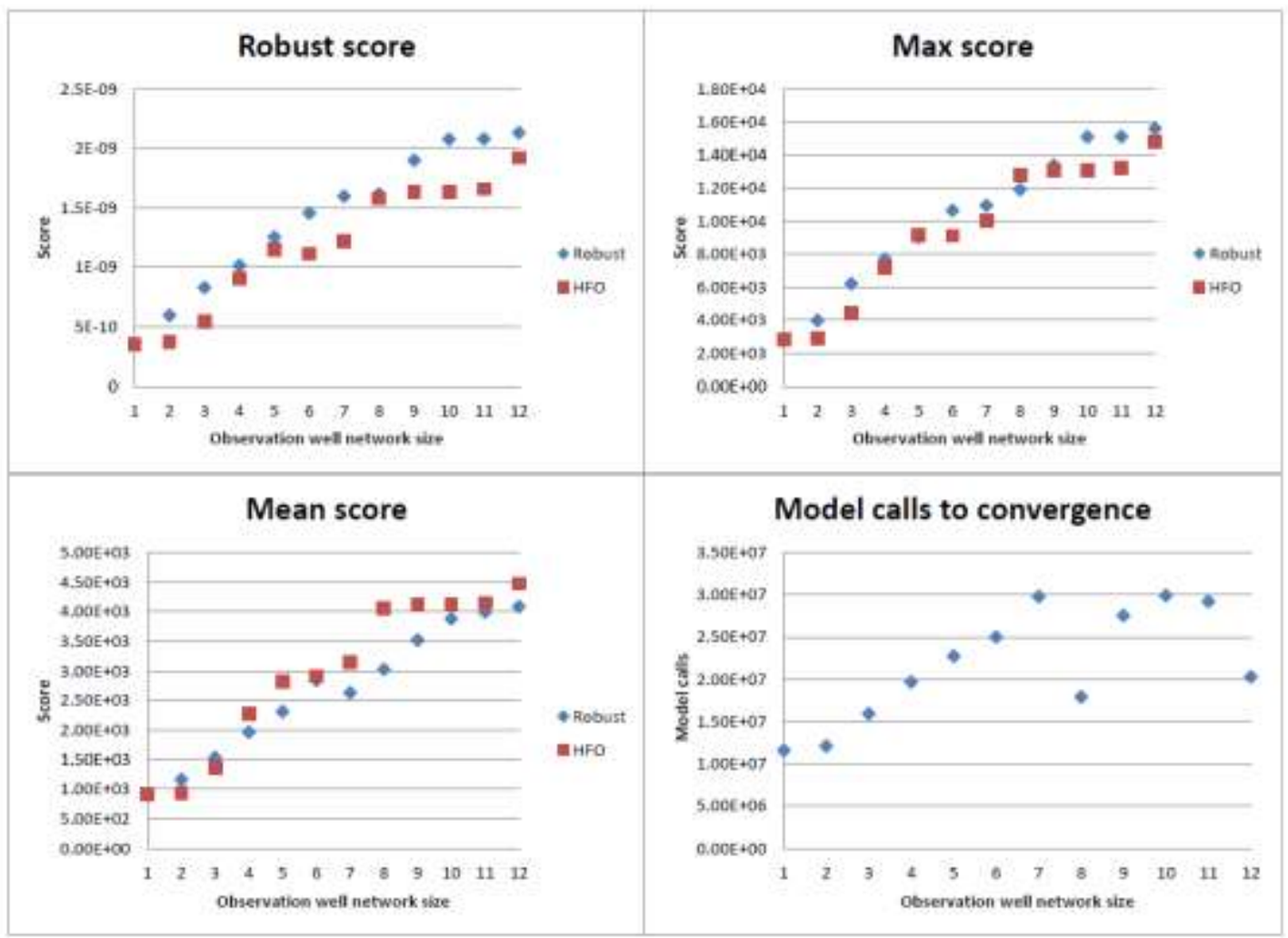

Figure 16. Results from the 2-D test case 
In Figures 9-11, contours represent the frequency at which a particular location is chosen as a $\tilde{\boldsymbol{\omega}}_{i}^{(j)}$ (the $j^{\text {th }}$ optimal location in $\tilde{\boldsymbol{\omega}}_{i}$ ). This frequency is scaled to add-up to one, giving us an estimated probability that a particular location will be a $\tilde{\boldsymbol{\omega}}_{i}^{(j)}$. As we see in the figures, as the dimension of the optimization problem (the number of observation wells being optimized) increases, the number of unique locations chosen as a $\tilde{\boldsymbol{\omega}}_{i}^{(j)}$ grows. However, the highest frequency locations tend to remain in the same general area of the aquifer. We observe in the figures that all $\tilde{\boldsymbol{\omega}}_{i}^{(j)}$ seem to show a higher preference for the observation zones in the upper left corner of the aquifer and a lower preference for the observation zones in the lower center of the aquifer. It is interesting to point-out that when we optimize the twelve observation well network almost every feasible location is chosen as a $\tilde{\boldsymbol{\omega}}_{i}^{(j)}$ at least once. Figures 12-14 show the HFO designs (x) and robust designs (०). From the figures, one sees that the HFO designs tend to be fairly close to the robust designs. However, only in one observation well network are the two designs the same. We compare the scores of the HFO and robust designs in the same way as in the 1-D test case -- the max-min (robust) score, the max score, and the mean score. Again, as with the 1-D test case, the results are tied closely to the specific experiment setup. For the 2-D 


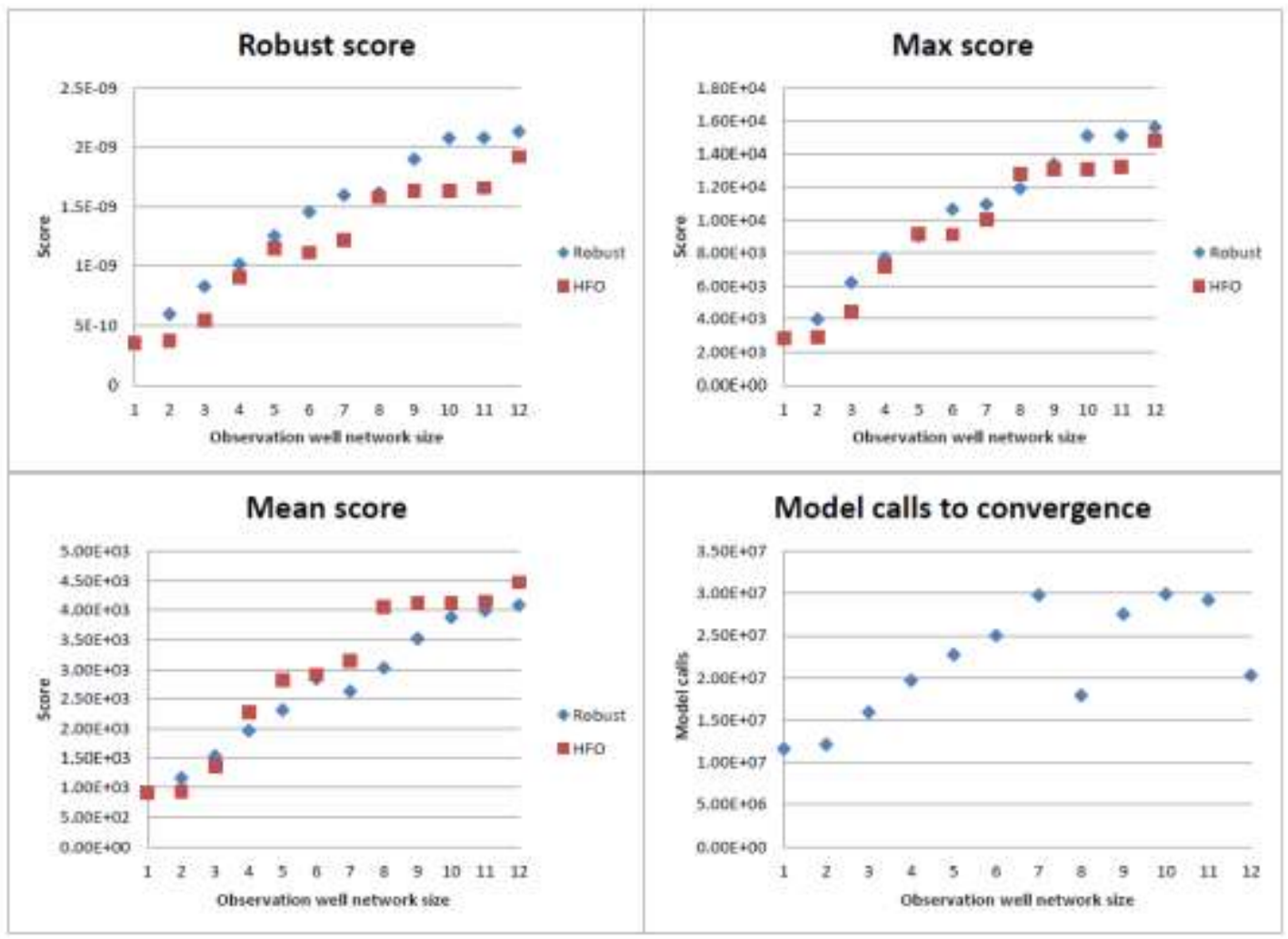

Figure 15 shows that the robust design outperforms the HFO design in robust score (as expected) and, for most cases, in max score. On the other hand, we see that for most cases the HFO design outperforms the robust design in mean score. In all cases, however, the scores are fairly close. As with the 1-D test case, the figures that are perhaps most interesting are the ones that show the contour plots estimating the probability of a location being an optimal location. Figure 15 also shows the number of model calls required by the algorithm to achieve convergence. As expected, in general as optimization complexity increases (i.e. larger observation well networks), the number of model calls required to achieve convergence increases. Since this is a heuristic algorithm, this is not an absolute requirement; thus we observe that for some optimizations, the algorithm converges faster than for less complex optimizations. 


\section{Conclusions}

We have developed a robust experimental design algorithm that can be used to select the locations for a network of observation wells that provide maximum robust information about the unknown hydraulic conductivities. The algorithm maximizes information in the worst-case scenario. The formulated combinatorial, max-min problem is nonlinear and non-convex with mixed integer variables. These characteristics make it very difficult, if not impossible, to solve a real-world-sized problem using traditional mathematical programming techniques, such as mixed integer nonlinear programming. This paper's novelty is demonstrating that a genetic algorithm (GA) coupled with a parameter-independent reduced model is able to overcome this difficulty.

The algorithm accomplishes this by taking some feasible range of hydraulic conductivity for each of the $n_{z}$ hydrologic zones with unknown hydraulic conductivity, discretizing this range into $n_{l}$ increments, and forming all $n_{l}^{n_{z}}$ possible combinations of these zonal hydraulic conductivities. Then, for a given number of observation wells $\left(n_{\text {obswell }}\right)$, a groundwater model approximates the Jacobian and information matrices to find $n_{l}^{n_{z}}$ solutions $\left(\tilde{\boldsymbol{\omega}}_{i, i=1, \ldots, n_{l}^{n_{z}}}\right)$, each of which maximizes the information criterion for a given parameter combination. The algorithm scores each feasible location with the number of times that location is selected as part of a $\tilde{\boldsymbol{\omega}}_{i}$ and creates a contour map of the score of each feasible location (scaled to sum to one). This approximates the probability of a feasible location serving as an optimal location (i.e. being a part of $\left.\omega^{*}\right)$. The $n_{\text {obswell }}$ locations with the highest scores are classified as the High Frequency Observation Well (HFO) design. The algorithm adds this design to the original set of designs $(\hat{\boldsymbol{\omega}})$ to produce a final set $\left(\hat{\boldsymbol{\omega}}_{+1}\right)$ with $n_{l}^{n_{z}}+1$ designs. A groundwater model then is called to 
evaluate the optimality (i.e. fitness score) of all designs in $\hat{\boldsymbol{\omega}}_{+1}$ for all $n_{l}^{n_{z}}$ parameter combinations. The algorithm records the robust, maximum, and mean scores for each design and classifies the design with the maximum-minimum score as the robust design. As we have shown, this algorithm requires an inordinately large number of groundwater model calls, rendering calling a full groundwater model infeasible. To overcome this, the algorithm calls a parameterindependent reduced groundwater model. The reduced model runs several orders of magnitude faster than the full model. This allows the problem to be solved in the reduced space, drastically reducing the computational burden of the algorithm.

We first tested our algorithm on a small 1-D experimental setup to test its convergence and then ran it on a realistically-sized 2-D experimental setup. The parameter-independent model reduction algorithm reduced the 303-equation, 1-D full model to a 25-equation reduced model and the 29,197-equation full 2-D model to a 113-equation reduced model. In both cases, because of model reduction, the algorithm achieved convergence in a reasonable amount of time.

For the two experimental setups, the HFO design performs similarly, but slightly worse than the robust design and sometimes outperforms the robust design in max and mean score. We find the estimated probability of a feasible location being an optimal location by plotting the frequency (scaled to sum to one) that a particular feasible location is selected as a $\tilde{\boldsymbol{\omega}}_{i}^{(j)}$ (the $j^{\text {th }}$ optimal location in $\tilde{\boldsymbol{\omega}}_{i}$ ). Executing this algorithm for the 1-D case took 761,817 model calls and $142,728,056$ model calls for the 2-D case. Given that we saw a time improvement of several orders of magnitude with both the 1-D and 2-D test cases, the advantage of solving the problem in the reduced space is obvious. For comparative perspective, if we were to use a GA coupled to 
the full 2-D model at 64 seconds per model call it would take 9E9 seconds, or 289.7 years, to achieve convergence. Clearly this is completely infeasible, but by replacing the full model with the reduced model we are able to solve this formerly infeasible experimental design problem.

For both the 1-D and 2-D cases the algorithm converged to robust and HFO designs that had similar scores. As expected, the robust design always performed at least as well as the HFO design in robust scoring. On the other hand, there seemed to be no real pattern controlling which design performed better with the mean or maximum scores. We expect that the maximum and mean scores are very sensitive to the experimental setup. Perhaps even more interesting than these results, the algorithm allows us to approximate the probability of a feasible location serving as an optimal location for both test cases. Along with solving for the robust and HFO designs, this approximation also would be infeasible without coupling the reduced model to the algorithm. The results show that the GA coupled with a parameter-independent reduced model is a valid search method to search for the optimal solution to the computationally expensive maxmin optimization required in robust experimental design. Along the way, the algorithm can collect additional data, such as finding the HFO design and mapping an approximation of the probability of a location being an optimal location.

In future work we could research experimental designs that seek information about both unknown parameters and unknown pumping rates. We also could apply parameter and pumpingindependent reduced modeling to study the data sufficiency problem of designing an optimal pump test and observation network. Another application that may be of interest would be 
coupling a reduced model to a conjunctive use optimization model, either with known or unknown parameters.

\section{Notation}

\begin{tabular}{|c|c|}
\hline $\boldsymbol{A} \in R^{N_{n} \times N_{n}}$ & Stiffness matrix for the full model \\
\hline$\tilde{\boldsymbol{A}} \in R^{N_{p} x N_{p}}$ & Stiffness matrix for the reduced model \\
\hline $\boldsymbol{A}(\boldsymbol{k}) \in R^{N_{n} x N_{n}}$ & Matrix representing the system of linear equations in the full model \\
\hline$\tilde{\boldsymbol{A}}(\boldsymbol{k}) \in R^{n_{p} \times n_{p}}$ & Matrix representing the system of linear equations in the reduced model \\
\hline $\boldsymbol{B} \in R^{N_{n} \times N_{n}}$ & Mass matrix for the full model \\
\hline $\boldsymbol{B} \in R^{n_{p} \times n_{p}}$ & Mass matrix for the reduced model \\
\hline $\boldsymbol{b} \in R^{N_{n}}$ & Vector describing the forcing of the full model \\
\hline$\tilde{\boldsymbol{b}} \in R^{N_{n}}$ & Vector describing the forcing of the reduced model \\
\hline$\chi$ & Matrix of snapshots collected at some hydraulic conductivity $\boldsymbol{k}$ \\
\hline$d$ & Scaling factor for the residual \\
\hline$F$ & Specific volumetric pumping rate \\
\hline$\Delta K_{j}$ & Perturbation of the $j^{\text {th }}$ hydraulic conductivity \\
\hline$\Delta \boldsymbol{k}_{j} \in R^{n_{z}}$ & Vector perturbing the $j^{\text {th }}$ hydraulic conductivity \\
\hline$\Delta o_{i}$ & Change in the $i^{t h}$ observation \\
\hline$\Delta \theta_{j}$ & Perturbation of the $j^{\text {th }}$ parameter \\
\hline$\Delta \boldsymbol{\theta}_{j} \in R^{N}$ & Vector perturbing the $j^{\text {th }}$ parameter \\
\hline$f_{1}, f_{2}, f_{3}$ & Known functions describing initial and boundary conditions of an aquifer \\
\hline $\boldsymbol{e}$ & Error between the full and reduced model \\
\hline$\Gamma_{1}$ & Fixed head boundary \\
\hline$\Gamma_{2}$ & Flux boundary \\
\hline $\boldsymbol{H} \in R^{N_{n} x n_{s p}}$ & Vector of initial head values \\
\hline $\boldsymbol{h} \in R^{N_{n} x n_{s p}}$ & Vector of head values \\
\hline $\mathrm{HFO}$ & High frequency observation well design \\
\hline $\boldsymbol{I} \in R^{n_{q} \times n_{q}}$ & Information matrix calculated by $\boldsymbol{J}_{d}{ }^{T} * \boldsymbol{J}_{d} * \boldsymbol{W}$ \\
\hline $\boldsymbol{J}_{d} \in R^{n_{o b s} \times \mathrm{n}_{q}}$ & Jacobian matrix containing only the observations of interest to all wells \\
\hline$J_{d_{i, j}}$ & $i, j^{\text {th }}$ element in $\boldsymbol{J}_{d}$ \\
\hline $\boldsymbol{K} \in R^{n_{z} \times n_{l}^{n_{z}}}$ & Matrix of columns storing all parameter combinations to be searched \\
\hline$K_{i}$ & Hydraulic conductivity in the $i^{\text {th }}$ direction \\
\hline$\hat{\boldsymbol{K}}$ & The space of feasible hydraulic conductivities \\
\hline $\boldsymbol{k} \in R^{n_{z}}$ & Vector of hydraulic conductivities \\
\hline $\boldsymbol{M} \in R^{n_{\text {obswell }} \times N_{n}}$ & Observation matrix isolating the rows of $\boldsymbol{P}$ corresponding to some $\boldsymbol{\omega}$ \\
\hline
\end{tabular}




\begin{tabular}{|c|c|}
\hline$\Lambda \in R^{N_{n} \times N_{n}}$ & Matrix containing the eigenvalues of $\boldsymbol{X}$ \\
\hline $\mathrm{L}$ & Length unit (meters, feet, etc.) \\
\hline$N$ & Total number of parameters of interest \\
\hline$n_{l}$ & Number of parameter increments \\
\hline$N_{n}$ & Number of nodes in the full model \\
\hline$n_{o b s}$ & Total number of observations taken \\
\hline$n_{\text {obswell }}$ & Maximum number of allowable observation wells \\
\hline$n_{p}$ & Number of principal components used in the reduced model \\
\hline$n_{s p}$ & Number of snapshots taken for each pumping well to build the reduced model \\
\hline$n_{z}$ & Number of hydrologic zones with unknown hydraulic conductivity \\
\hline $\boldsymbol{P} \in R^{N_{n} \times n_{p}}$ & Full projection matrix \\
\hline$\hat{\boldsymbol{P}} \in R^{N_{n} \times n_{s p}}$ & Principle vectors of a particular set of snapshots \\
\hline PDE & Partial differential equation \\
\hline $\boldsymbol{\Omega}$ & The space of all feasible sets of observation well locations \\
\hline$\omega \in R^{n_{o b s w e l l}}$ & Vector contacting some feasible set of observation well locations $(\boldsymbol{\omega} \subset \boldsymbol{\Omega})$ \\
\hline$\hat{\boldsymbol{\omega}} \in R^{n_{o b s} \times n_{l}^{n_{z}}}$ & The set of all $\tilde{\boldsymbol{\omega}}_{i}$ \\
\hline$\hat{\boldsymbol{\omega}}_{+1} \in R^{n_{o b s} \times n_{l}^{n_{z}+1}}$ & $\hat{\boldsymbol{\omega}}$ plus the HFO design \\
\hline$\omega^{*} \in R^{n_{o b s w e l l}}$ & The optimal experimental design solution given the true aquifer parameters \\
\hline$\tilde{\boldsymbol{\omega}}_{i} \in R^{n_{\text {obswell }}}$ & $i^{t h}$ solution output by the GA \\
\hline$\tilde{\boldsymbol{\omega}}_{i}^{(j)}$ & $j^{\text {th }}$ location in $\tilde{\boldsymbol{\omega}}_{i}$ \\
\hline ODE & Ordinary differential equation \\
\hline$o_{i}$ & $i^{\text {th }}$ observation \\
\hline$o_{i}(\boldsymbol{\theta})$ & $i^{\text {th }}$ model simulated value using the parameter values in $\boldsymbol{\theta}$ \\
\hline$Q$ & Matrix containing the eigenvectors of $\boldsymbol{X}$ \\
\hline $\boldsymbol{q} \in R^{N_{n}}$ & Vector of sinks for the full model \\
\hline$q_{n}$ & Specific discharge normal to the flux boundary $\left(\Gamma_{2}\right)$ \\
\hline $\boldsymbol{R}(\boldsymbol{k}) \in R^{n_{p} x n_{o b s}}$ & Matrix containing the reduced solution at the observation times \\
\hline $\boldsymbol{r} \in R^{n_{p}}$ & Vector of the reduced solution at time $t$ \\
\hline$r_{e}$ & The residual between the full and reduced model \\
\hline $\boldsymbol{s} \in R^{N_{n} \times n_{s p}}$ & Vector of drawdown values \\
\hline$S_{i}$ & $i^{\text {th }}$ drawdown value \\
\hline $\boldsymbol{\Sigma} \in R^{n_{p} x n_{p}}$ & Diagonal matrix containing the singular values of $\boldsymbol{X}$ \\
\hline$S_{s}$ & Specific storage \\
\hline$\hat{\boldsymbol{S}}_{t} \in R^{N_{n}}$ & Vector of the approximation of $s_{t}$ \\
\hline SVD & Singular vale decomposition \\
\hline $\mathrm{T}$ & Time unit (days, hours, etc.) \\
\hline$\tau$ & Error tolerance \\
\hline
\end{tabular}




\begin{tabular}{|cl|}
\hline $\boldsymbol{\theta} \in R^{N}$ & Vector of nominal parameter values \\
$\theta_{j}$ & $j^{\text {th }}$ parameter \\
$V \subset R^{N_{n}}$ & Subspace spanned by the columns of $\boldsymbol{P}$ \\
$\boldsymbol{V} \in R^{n_{p}} x n_{p}$ & Matrix containing the right singular vectors of $\boldsymbol{X}$ \\
$\boldsymbol{W} \in R^{n_{q} x n_{q}}$ & Some user specified weighting matrix used in calculating $\boldsymbol{I}$ \\
$\boldsymbol{X} \in R^{N_{n}} \times n_{p}$ & Matrix containing all collected snapshots \\
$\boldsymbol{x} \in R^{N_{n}}$ & Vector of binary variables indicating if a node has an observation well \\
$x_{i}$ & $i^{\text {th }}$ element in $\boldsymbol{x}$ \\
$z$ one $j$ & All the nodes in the $j^{\text {th }}$ observation zone \\
\hline
\end{tabular}

\section{Acknowledgements}

This material is based on work supported by NSF under award EAR-1314422. Partial support also was provided by an AECOM endowment. 


\section{References}

[1] Reed P, Minsker B, Valocchi AJ. Cost-effective long-term groundwater monitoring design using a genetic algorithm and global mass interpolation. Water Resour Res 2000;36:373141 .

[2] McPhee J, Yeh WW-G. Experimental design for groundwater modeling and management. Water Resour Res 2006;42.

[3] Babbar-Sebens M, Minsker B. A Case-Based Micro Interactive Genetic Algorithm (CBMIGA) for interactive learning and search: Methodology and application to groundwater monitoring design. Environ Model Softw 2010;25:1176-87.

[4] Ushijima TT, Yeh WW-G. Experimental design for estimating unknown groundwater pumping using genetic algorithm and reduced order model. Water Resour Res 2013;49:6688-99.

[5] Bear J. Hydraulics of Groundwater. London: McGraw-Hill; 1979.

[6] Cazemier W, Verstappen RWCP, Veldman a. EP. Proper orthogonal decomposition and low-dimensional models for driven cavity flows. Phys Fluids 1998;10:1685-99.

[7] Willcox K, Peraire J. Balanced Model Reduction via the Proper Orthogonal Decomposition. AIAA J 2002;40:2323-30.

[8] Kowalski ME, Jin J-M. Model-order reduction of nonlinear models of electromagnetic phased-array hyperthermia. IEEE Trans Biomed Eng 2003;50:1243-54.

[9] Vermeulen PTM, Heemink AW, Te Stroet CBM. Reduced models for linear groundwater flow models using empirical orthogonal functions. Adv Water Resour 2004;27:57-69.

[10] McPhee J, Yeh WW-G. Groundwater Management Using Model Reduction via Empirical Orthogonal Functions. J Water Resour Plan Manag 2008;134:161-70.

[11] Siade AJ, Putti M, Yeh WW-G. Snapshot selection for groundwater model reduction using proper orthogonal decomposition. Water Resour Res 2010;46:W08539.

[12] Siade AJ, Putti M, Yeh WW-G. Reduced order parameter estimation using quasilinearization and quadratic programming. Water Resour Res 2012;48:W06502.

[13] Pasetto D, Putti M, Yeh WW-G. A reduced-order model for groundwater flow equation with random hydraulic conductivity: Application to Monte Carlo methods. Water Resour Res 2013;49:3215-28. 
[14] Grepl MA, Patera AT. A posteriori error bounds for reduced-basis approximations of parametrized parabolic partial differential equations. ESAIM Math Model Numer Anal 2005;39:157-81.

[15] Rovas D V., Machiels L, Maday Y. Reduced-basis output bound methods for parabolic problems. IMA J Numer Anal 2005;26:423-45.

[16] Haasdonk B, Ohlberger M. Efficient reduced models and a posteriori error estimation for parametrized dynamical systems by offline/online decomposition. Math Comput Model Dyn Syst 2011;17:145-61.

[17] Hasenauer J, Löhning M, Khammash M, Allgöwer F. Dynamical optimization using reduced order models: A method to guarantee performance. J Process Control 2012;22:1490-501.

[18] Hinze M, Kunkel M. Residual based sampling in POD model order reduction of driftdiffusion equations in parametrized electrical networks. ZAMM - J Appl Math Mech / Zeitschrift Für Angew Math Und Mech 2012;92:91-104.

[19] Shlizerman E, Ding E, Williams MO, Kutz JN. The Proper Orthogonal Decomposition for Dimensionality Reduction in Mode-Locked Lasers and Optical Systems. Int J Opt 2012;2012:1-18.

[20] Yeh WW-G. Review of Parameter Identification Procedures in Groundwater Hydrology: The Inverse Problem. Water Resour Res 1986;22:95-108.

[21] Poeter EP, Hill MC, Banta ER, Mehl S, Chirstensen S. UCODE 2005 and Six Other Comptuer Codes for Universal Sensitivity Analysis, Calibration, and Uncertainty Evaluation. US Geol Surv Tech Methods 2005;6-A11:283p.

[22] Cleveland TG, Yeh WW-G. Sampling Network Design for Transport Parameter Identification. J Water Resour Plan Manag 1990;116:764-83.

[23] Kutner MH, Nachtsheim CJ, Li W. Applied Linear Statistical Models. 5th ed. New York: McGraw-Hill; 2004.

[24] Steinberg DM, Hunter WG. Experimental Design: Review and Comment. Technometrics 1984;26:71-97.

[25] Hsu N-S, Yeh WW-G. Optimum experimental design for parameter identification in groundwater hydrology. Water Resour Res 1989;25:1025-40.

[26] Altmann-Dieses, Schloder JP, Bock HG, Richter O. Optimal experimental design for parameter estimation in column outflow experiments. Water Resour Res 2002;38:1186. 
[27] Knopman DS, Voss CI. Multiobjective sampling design for parameter estimation and model discrimination in groundwater solute transport. Water Resour Res 1989;25:224558 .

[28] Nishikawa T, Yeh WW-G. Optimal pumping test design for the parameter identification of groundwater systems. Water Resour Res 1989;25:1737-47.

[29] Catania F, Paladino O. Optimal sampling for the estimation of dispersion parameters in soil columns using an Iterative Genetic Algorithm. Environ Model Softw 2009;24:115-23.

[30] Cleveland TG, Yeh WW -G. Optimal Configuration and Scheduling of Ground-Water Tracer Test. J Water Resour Plan Manag 1991;117:37-51.

[31] McCarthy JM, Yeh WW-G. Optimal pumping test design for parameter estimation and prediction in groundwater hydrology. Water Resour Res 1990;26:779-91.

[32] Chang L-F, Sun N-Z, Yeh WW-G. Optimal observation network design for parameter structure identification in groundwater modeling. Water Resour Res 2005;41:W03002.

[33] Dietrich F, Raatz A, Hesselbach J. A-priori Fisher information of nonlinear state space models for experiment design. 2010 IEEE Int. Conf. Robot. Autom., IEEE; 2010, p. 3698-702.

[34] De Brauwere A, De Ridder F, Gourgue O, Lambrechts J, Comblen R, Pintelon R, et al. Design of a sampling strategy to optimally calibrate a reactive transport model: Exploring the potential for Escherichia coli in the Scheldt Estuary. Environ Model Softw 2009;24:969-81.

[35] Sun N-Z, Yeh WW-G. Development of objective-oriented groundwater models: 2. Robust experimental design. Water Resour Res 2007;43:W02421.

[36] Wall M. GAlib: A C++ Library of Genetic Algorithm Components. galib247 1995:1-104.

[37] Paniconi C, Putti M. A comparison of Picard and Newton iteration in the numerical solution of multidimensional variably saturated flow problems. Water Resour Res 1994;30:3357-74.

[38] Cau PL, Lecca G, Putti M, Paniconi C. The influence of a confining layer on saltwater intrusion under surface recharge and groundwater extraction conditions. Comput Methods Water Resour Dev Water Resour 2002:493-500. 